\title{
Sparse Coding on Symmetric Positive Definite Manifolds using Bregman Divergences
}

\author{
Mehrtash Harandi, Richard Hartley, Brian Lovell, Conrad Sanderson
}

\begin{abstract}
This paper introduces sparse coding and dictionary learning for Symmetric Positive Definite (SPD) matrices, which are often used in machine learning, computer vision and related areas. Unlike traditional sparse coding schemes that work in vector spaces, in this paper we discuss how SPD matrices can be described by sparse combination of dictionary atoms, where the atoms are also SPD matrices. We propose to seek sparse coding by embedding the space of SPD matrices into Hilbert spaces through two types of Bregman matrix divergences. This not only leads to an efficient way of performing sparse coding, but also an online and iterative scheme for dictionary learning. We apply the proposed methods to several computer vision tasks where images are represented by region covariance matrices. Our proposed algorithms outperform state-of-the-art methods on a wide range of classification tasks, including face recognition, action recognition, material classification and texture categorization.
\end{abstract}

Index Terms-Riemannian geometry, Bregman divergences, kernel methods, sparse coding, dictionary learning.

\section{INTRODUCTION}

Sparsity is a popular concept in signal processing [13], [39], [59] and stipulates that natural signals like images can be efficiently described using only a few non-zero coefficients of a suitable basis (i.e. dictionary) [13]. This paper introduces techniques to perform sparse coding on Symmetric Positive Definite (SPD) matrices. More specifically, unlike traditional sparse coding schemes that work on vectors, in this paper we discuss how SPD matrices can be described by sparse combination of dictionary atoms, where the atoms are also SPD matrices.

Our motivation stems from pervasive role of SPD matrices in machine learning, computer vision and related areas. For example, SPD matrices have been used in medical imaging, texture classification [21], [54], action recognition and gesture categorization [45], as well as face recognition [21], [40].

Extending sparse coding methods to SPD matrices is not trivial, since such matrices form the interior of the positive semidefinite cone. In other words, simply vectorizing SPD matrices and employing Euclidean geometry (e.g., Euclidean norms) does not lead to accurate representations [27], [41], [55]. To overcome the drawbacks of Euclidean structure, SPD matrices are usually analyzed using a Riemannian structure, known as SPD or tensor manifold [41]. This is where

\footnotetext{
Published in: IEEE Trans. Neural Networks and Learning Systems, (in press).

Mehrtash Harandi and Richard Hartley are with the College of Engineering and Computer Science, Australian National University, and with NICTA, Australia. Brian Lovell is with the University of Queensland, Australia. Conrad Sanderson is with NICTA, Australia, and the University of Queensland, Australia.
}

the difficulties arise. On one hand, taking into account the Riemannian geometry is important as discussed in various recent studies [21], [27], [41], [55]. On the other hand, the non-linearity of the Riemannian structure is a hindrance and demands specialized machineries.

Generally speaking, two approaches to handle the nonlinearity of Riemannian manifolds are (i) locally flattening them via tangent spaces [45], [55], and (ii) embedding them in higher dimensional Hilbert spaces [7], [21], [27]. The latter has recently received a surge of attention, since embedding into Reproducing Kernel Hilbert Space (RKHS) through kernel methods [49] is a well-established and principled approach in machine learning. However, embedding SPD manifolds into RKHS requires non-trivial kernel functions defined on such manifolds, which, according to Mercer's theorem [49], must be positive definite.

The contributions in this paper ${ }^{1}$ are four-fold:

(i) We propose sparse coding and dictionary learning algorithms for data points (matrices) on SPD manifolds, by embedding the manifolds into RKHS. This is advantageous, as linear geometry applies in RKHS.

(ii) For the embedding we propose kernels derived from two Bregman matrix divergences, namely the Stein and Jeffrey divergences. While the kernel property of the Jeffrey divergence was discovered in 2005 [23], to our best knowledge, this is one of the first attempts to benefit from this kernel for analyzing SPD matrices.

(iii) For both kernels, we devise a closed-form solution for updating an SPD dictionary atom by atom.

(iv) We apply the proposed methods to several computer vision tasks where images are represented by region covariance matrices. Our proposed algorithms outperform state-of-the-art methods on several classification tasks, including face recognition, texture classification and action recognition.

\section{RELATED WORK}

In computer vision, SPD matrices are used in various applications, including pedestrian detection [55], texture classification [21], [54], object recognition [27], object tracking [54], action recognition [18], [45] and face recognition [21], [40]. This is mainly because Region Covariance Descriptors (RCM) [54], which encode second order statistics, are straightforward and relatively robust descriptors for images and videos. Moreover, structure tensors, which are by nature

\footnotetext{
${ }^{1}$ This paper is a thoroughly extended and revised version of our earlier work [21]. In addition to providing more insights on the proposed methods, we extend our primary ideas by studying and devising coding and dictionary learning methods in the RKHS induced by the Jeffrey kernel. We also devise an efficient algorithm to obtain sparse codes in our RKHS-based formulation.
} 
SPD matrices, encode important image features (e.g., texture and motion in optical flow estimation and motion segmentation). Lastly, diffusion tensors that naturally arise in medical imaging are described by $3 \times 3$ SPD matrices [41].

Our interest in this paper is to perform sparse coding and dictionary learning on SPD matrices, since modern systems in various applications benefit from the notion of sparse coding. However, while significant steps have been taken to develop the theory of the sparse coding and dictionary learning in Euclidean spaces, only a handful of studies tackle similar problems for SPD matrices [18], [25], [53].

Sra and Cherian [53] proposed to measure the similarity between SPD matrices using the Frobenius norm and formulated the sparse coding and dictionary learning problems accordingly. While solving the problems using purely Euclidean structure of SPD matrices is computationally attractive, it neglects the Riemannian structure of SPD manifolds.

A somehow similar and straightforward idea is to flatten an SPD manifold using a fixed tangent space. Sparse coding by embedding manifolds into their identity tangent spaces, which identifies the Lie algebra of SPD manifolds, is considered in [15], [18], [62]. Though such embedding considerably simplifies the sparse coding formulation, the pair-wise distances are no longer adequate, which can affect discrimination performance. This is exacerbated for manifolds with negative curvature (e.g. SPD manifolds), since pair-wise distances are not even directly bounded ${ }^{2}$.

A more involved approach to learn a Riemannian dictionary is proposed very recently by Ho et al. [25]. The underlying idea is to exploit the tangent bundle of the manifold. To avoid a trivial solution in this approach, an affine constraint has to be added to the general formulation [25]. While this results in independency to the origin, it no longer addresses the original problem. Furthermore, switching back and forth to tangent spaces of SPD manifolds (as required by this formulation) can be computationally very demanding for high dimensional manifolds.

Sivalingam et al. [50], [51] proposed Tensor Sparse Coding (TSC) which utilizes the Burg divergence (an asymmetric type of Bregman divergence) to perform sparse coding and dictionary learning on SPD manifolds. To this end, they show that when the Burg divergence is used as the proximity measure, the problem of sparse coding becomes a MAXDET problem which is convex and hence can be solved by interior point algorithms [50]. As for dictionary learning, two methods were proposed in [50], [51]. In the first method, a gradient descent approach was utilized to update dictionary atoms one by one. Inspired by the K-SVD algorithm [1], the second method updates dictionary atoms by minimizing a form of residual error over training data, which speeds up the process of dictionary learning. Besides the asymmetric nature of the Burg divergence, we note that the computational complexity of the TSC algorithm is high, especially for high-dimensional SPD manifolds.

\footnotetext{
${ }^{2}$ For manifolds with positive curvature, pair-wise distances on tangent spaces are greater or equal to true geodesic distances on the manifold according to Toponogov's theorem [32]. Such property does not hold for manifolds with negative curvature.
}

\section{PReliminaries}

This section provides an overview on Riemannian geometry of SPD manifolds, Bregman divergences and their properties. It provides the groundwork for techniques described in following sections. Throughout the paper, bold capital letters denote matrices (e.g., $\boldsymbol{X})$ and bold lower-case letters denote column vectors $(e . g ., \boldsymbol{x})$. Notation $x_{i}$ is used to indicate element at position $i$ of vector $\boldsymbol{x}$. $\mathbf{I}_{n}$ is the $n \times n$ identity matrix. $\|\boldsymbol{x}\|_{2}=\sqrt{\boldsymbol{x}^{T} \boldsymbol{x}}$ and $\|\boldsymbol{x}\|_{1}=\sum_{i}\left|x_{i}\right|$ denote the $\ell_{2}$ and $\ell_{1}$ norms, respectively, with $T$ indicating matrix transpose. $\|\boldsymbol{X}\|_{F}=\sqrt{\operatorname{Tr}}\left(\boldsymbol{X}^{T} \boldsymbol{X}\right)$ designates the Frobenius norm. $\mathrm{GL}(n)$ denotes the general linear group, the group of real invertible $n \times n$ matrices. $\operatorname{Sym}(n)$ is the space of real $n \times n$ symmetric matrices.

\section{A. Riemannian Geometry of SPD Manifolds}

An $n \times n$, real SPD matrix $\boldsymbol{X}$ has the property that $\boldsymbol{v}^{T} \boldsymbol{X} \boldsymbol{v}>$ 0 for all non-zero $\boldsymbol{v} \in \mathbb{R}^{n}$. The space of $n \times n$ SPD matrices, denoted by $\mathcal{S}_{++}^{n}$, is not a vector space since multiplying an SPD matrix by a negative scalar results in a matrix which does not belong to $\mathcal{S}_{++}^{n}$. Instead, $\mathcal{S}_{++}^{n}$ forms the interior of a convex cone in the $n(n+1) / 2$-dimensional Euclidean space. The $\mathcal{S}_{++}^{n}$ space is mostly studied when endowed with a Riemannian metric and thus forms a Riemannian manifold [41].

On a Riemannian manifold, a natural way to measure nearness is through the notion of geodesics, which are curves analogous to straight lines in $\mathbb{R}^{n}$. The geodesic distance is thus defined as the length of the shortest curve connecting the two points. The tangent space at a point $\boldsymbol{P}$ on the manifold, $T_{\boldsymbol{P}} \mathcal{M}$, is a vector space that consists of the tangent (i.e., velocity) vectors of all possible curves passing through $\boldsymbol{P}$.

Two operators, namely the exponential map $\exp _{\boldsymbol{P}}(\cdot)$ : $T_{\boldsymbol{P}} \mathcal{M} \rightarrow \mathcal{M}$ and the logarithm map $\log _{\boldsymbol{P}}(\cdot)=\exp _{\boldsymbol{P}}^{-1}(\cdot):$ $\mathcal{M} \rightarrow T_{P} \mathcal{M}$, are defined over Riemannian manifolds to switch between the manifold and tangent space at $\boldsymbol{P}$. The exponential operator maps a tangent vector $\Delta$ to a point $\boldsymbol{X}$ on the manifold. The property of the exponential map ensures that the length of $\Delta$ becomes equal to the geodesic distance between $\boldsymbol{X}$ and $\boldsymbol{P}$. The logarithm map is the inverse of the exponential map, and maps a point on the manifold to the tangent space $T_{\boldsymbol{P}}$. The exponential and logarithm maps vary as point $\boldsymbol{P}$ moves along the manifold.

On the SPD manifold, the Affine Invariant Riemannian Metric (AIRM) [41], defined as:

$$
\begin{aligned}
\langle\boldsymbol{V}, \boldsymbol{W}\rangle_{\boldsymbol{P}} & \triangleq\left\langle\boldsymbol{P}^{-1 / 2} \boldsymbol{V} \boldsymbol{P}^{-1 / 2}, \boldsymbol{P}^{-1 / 2} \boldsymbol{W} \boldsymbol{P}^{-1 / 2}\right\rangle \\
& =\operatorname{Tr}\left(\boldsymbol{P}^{-1} \boldsymbol{V} \boldsymbol{P}^{-1} \boldsymbol{W}\right)
\end{aligned}
$$

for $\boldsymbol{P} \in \mathcal{S}_{++}^{n}$ and $\boldsymbol{V}, \boldsymbol{W} \in T_{\boldsymbol{P}} \mathcal{M}$, induces the following geodesic distance between points $\boldsymbol{X}$ and $\boldsymbol{Y}$ :

$$
\delta_{R}(\boldsymbol{X}, \boldsymbol{Y})=\left\|\log \left(\boldsymbol{X}^{-1 / 2} \boldsymbol{Y} \boldsymbol{X}^{-1 / 2}\right)\right\|_{F},
$$

with $\log (\cdot)$ being the principal matrix logarithm. 


\section{B. Bregman Divergences}

In this part we introduce two divergences derived from Bregman matrix divergence, namely the Jeffrey and Stein divergences. We discuss their properties and establish their relations to AIRM. This provides motivation and grounding for our formulation of sparse coding and dictionary learning using the aforementioned divergences.

Definition 1. Let $\zeta: \mathcal{S}_{++}^{n} \rightarrow \mathbb{R}$ be a strictly convex and differentiable function defined on the symmetric positive cone $\mathcal{S}_{++}^{n}$. The Bregman matrix divergence $d_{\zeta}: \mathcal{S}_{++}^{n} \times \mathcal{S}_{++}^{n} \rightarrow$ $[0, \infty)$ is defined as

$$
d_{\zeta}(\boldsymbol{X}, \boldsymbol{Y})=\zeta(\boldsymbol{X})-\zeta(\boldsymbol{Y})-\left\langle\nabla_{\zeta}(\boldsymbol{Y}), \boldsymbol{X}-\boldsymbol{Y}\right\rangle,
$$

where $\langle\boldsymbol{X}, \boldsymbol{Y}\rangle=\operatorname{Tr}\left(\boldsymbol{X}^{T} \boldsymbol{Y}\right)$, and $\nabla_{\zeta}(\boldsymbol{Y})$ represents the gradient of $\zeta$ evaluated at $\boldsymbol{Y}$.

Loosely speaking, the Bregman divergence between $\boldsymbol{X}$ and $\boldsymbol{Y}$ can be understood as the distance between the function $\zeta(\boldsymbol{X})$ and its first order Taylor approximation constructed at $\boldsymbol{Y}$. The Bregman divergence is asymmetric, non-negative, and definite (i.e., $d_{\zeta}(\boldsymbol{X}, \boldsymbol{Y})=0$, iff $\boldsymbol{X}=\boldsymbol{Y}$ ). While the Bregman divergence enjoys a variety of useful properties [29], its asymmetric behavior can be a hindrance (e.g., in SVMs, the kernels need to be symmetric, hence asymmetric divergences cannot be used to devise kernels). In this paper we are interested in two types of symmetrized Bregman divergences, namely the Jeffrey and the Stein divergences.

Definition 2. The $J$ divergence (also known as Jeffrey or symmetric $K L$ divergence) is obtained from the Bregman divergence of Eqn. (3) by using $\zeta(\boldsymbol{X})=-\log |\boldsymbol{X}|$ as the seed function where $|\cdot|$ denotes determinant:

$$
\begin{aligned}
J(\boldsymbol{X}, \boldsymbol{Y}) & \triangleq \frac{1}{2} d_{\zeta}(\boldsymbol{X}, \boldsymbol{Y})+\frac{1}{2} d_{\zeta}(\boldsymbol{Y}, \boldsymbol{X}) \\
& =\frac{1}{2} \operatorname{Tr}\left(\boldsymbol{X}^{-1} \boldsymbol{Y}\right)-\frac{1}{2} \log \left|\boldsymbol{X}^{-1} \boldsymbol{Y}\right| \\
& +\frac{1}{2} \operatorname{Tr}\left(\boldsymbol{Y}^{-1} \boldsymbol{X}\right)-\frac{1}{2} \log \left|\boldsymbol{Y}^{-1} \boldsymbol{X}\right|-n \\
& =\frac{1}{2} \operatorname{Tr}\left(\boldsymbol{X}^{-1} \boldsymbol{Y}\right)+\frac{1}{2} \operatorname{Tr}\left(\boldsymbol{Y}^{-1} \boldsymbol{X}\right)-n .
\end{aligned}
$$

Definition 3. The Stein or $S$ divergence (also known as Jensen-Bregman LogDet divergence [10]) is obtained from the Bregman divergence of Eqn. (3) by again using $\zeta(\boldsymbol{X})=$ $-\log |\boldsymbol{X}|$ as the seed function but through Jensen-Shannon symmetrization:

$$
\begin{aligned}
S(\boldsymbol{X}, \boldsymbol{Y}) & \triangleq \frac{1}{2} d_{\zeta}\left(\boldsymbol{X}, \frac{\boldsymbol{X}+\boldsymbol{Y}}{2}\right)+\frac{1}{2} d_{\zeta}\left(\boldsymbol{Y}, \frac{\boldsymbol{X}+\boldsymbol{Y}}{2}\right) \\
& =\log \left|\frac{\boldsymbol{X}+\boldsymbol{Y}}{2}\right|-\frac{1}{2} \log |\boldsymbol{X} \boldsymbol{Y}|
\end{aligned}
$$

\section{Properties of $J$ and $S$ divergences}

The $J$ and $S$ divergences have a variety of properties which are akin to those of AIRM. The pertinent properties which inspired us to seek sparse coding on $\mathcal{S}_{++}^{n}$ using such divergences are:
- Both the $\mathbf{J}$ and $\mathbf{S}$ divergences as well as AIRM (as its name implies) are invariant to affine transformation [41], [52], [58].

- the length of curves under AIRM and S divergence is equal up to a scale [20].

- the geometric mean of two tensors under AIRM coincides with the geometric mean under $\mathrm{J}$ and $\mathrm{S}$ divergences (see [52] for the $\mathrm{S}$ divergence and the appendix for the proof on the $\mathrm{J}$ divergence).

The key message worth noting is the Hilbert space embedding property of the $J$ and $S$ divergences, which does not hold for AIRM [21], [27].

Hilbert space embedding (SPD kernels): Both $J$ and $S$ divergences admit a Hilbert space embedding in the form of a Radial Basis Function (RBF) kernel [49]. More specifically, for the $J$-divergence it has been shown that the kernel

$$
k_{J}(\boldsymbol{X}, \boldsymbol{Y})=\exp \{-\beta J(\boldsymbol{X}, \boldsymbol{Y})\}
$$

is conditionally positive definite [23]. Formally:

Definition 4 (Conditionally Positive Definite Kernels). Let $\mathcal{X}$ be a nonempty set. A symmetric function $\psi: \mathcal{X} \times \mathcal{X} \rightarrow \mathbb{R}$ is a conditionally positive definite kernel on $\mathcal{X}$ if and only if $\sum_{i, j=1}^{n} c_{i} c_{j} k\left(x_{i}, x_{j}\right) \geq 0$ for any $n \in \mathbb{N}, x_{i} \in \mathcal{X}$ and $c_{i} \in \mathbb{R}$ with $\sum_{i=1}^{n} c_{i}=0$.

The relations between positive definite $(p d)$ and conditionally positive definite $(c p d)$ kernels are studied by Berg et al. [3] and Schölkopf [46]. An important property of cpd kernels is

Proposition 1. For a kernel algorithm that is translation invariant, cpd kernels can be used instead of pd kernels [46].

This property relaxes the requirement of having $p d$ kernels for certain types of kernel algorithms. For example, in SVMs, a cpd kernel can be seamlessly used instead of a $p d$ kernel. We note that in [36] the kernel $k_{J}(\cdot, \cdot)$ was claimed to be positive definite. However, a formal proof is not available according to our best knowledge. For the Stein divergence, the kernel

$$
k_{S}(\boldsymbol{X}, \boldsymbol{Y})=\exp \{-\beta S(\boldsymbol{X}, \boldsymbol{Y})\},
$$

is guaranteed to be positive definite for

$$
\beta \in\left\{\frac{1}{2}, \frac{2}{2}, \cdots, \frac{n-1}{2}\right\} \cup\left\{\tau \in \mathbb{R}: \tau>\frac{1}{2}(n-1)\right\} .
$$

Interested reader is referred to [52] for further details. For values of $\beta$ outside of the above set, it is possible to convert a pseudo kernel into a true kernel, as discussed for example in [9].

\section{Sparse Coding}

Given a query $\boldsymbol{x} \in \mathbb{R}^{d}$, sparse coding in vector spaces optimizes the objective function

$$
l_{E}(\boldsymbol{x}, \mathbb{D}) \triangleq \min _{\boldsymbol{y}}\left\|\boldsymbol{x}-\sum_{j=1}^{N} y_{j} \boldsymbol{d}_{j}\right\|_{2}^{2}+\mathrm{Sp}(\boldsymbol{y}),
$$

with $\mathbb{D}_{d \times N}=\left[\boldsymbol{d}_{1}\left|\boldsymbol{d}_{2}\right| \cdots \mid \boldsymbol{d}_{N}\right], \boldsymbol{d}_{i} \in \mathbb{R}^{d}, N>d$ being a dictionary of size $N$. The function $\operatorname{Sp}(\boldsymbol{y})$ penalizes the 
solution if it is not sparse. The most common form of $l_{E}(\boldsymbol{x}, \mathbb{D})$ in the literature is obtained via $\ell_{1}$-norm regularization:

$$
l_{E}(\boldsymbol{x}, \mathbb{D}) \triangleq \min _{\boldsymbol{y}}\left\|\boldsymbol{x}-\sum_{j=1}^{N} y_{j} \boldsymbol{d}_{j}\right\|_{2}^{2}+\lambda\|\boldsymbol{y}\|_{1} .
$$

As elaborated in [19], directly translating the sparse coding problem to a non-flat Riemannian manifold $\mathcal{M}$ with a metric $\|\cdot\|_{\mathcal{M}}$ (such as geodesic distance) leads to re-writing Eqn. (10) as:

$$
l_{\mathcal{M}}(\boldsymbol{X}, \mathbb{D}) \triangleq \min _{\boldsymbol{y}}\left\|\boldsymbol{X} \ominus \biguplus_{j=1}^{N} y_{j} \odot \boldsymbol{D}_{j}\right\|_{\mathcal{M}}^{2}+\lambda\|\boldsymbol{y}\|_{1},
$$

where $\mathbb{D}=\left\{\boldsymbol{D}_{i}\right\}_{i=1}^{N}, \boldsymbol{D}_{i} \in \mathcal{M}$ is a Riemannian dictionary and $\boldsymbol{X} \in \mathcal{M}$ is a query point. The operators $\ominus, \biguplus$ and $\odot$ are Riemannian replacements for subtraction, summation and scalar multiplication, respectively. We note that the operators $\ominus$ and $\biguplus$ should be commutative and associative.

There are several difficulties in solving Eqn. (11). For example, metrics on Riemannian manifolds do not generally result in Eqn. (11) being convex [19]. As such, instead of solving Eqn. (11), here we propose to side-step the difficulties by embedding the manifold $\mathcal{M}$ into a Hilbert space $\mathcal{H}$ and replacing the idea of "combination" on manifolds with the general concept of linear combination in Hilbert spaces.

For the SPD manifold $\mathcal{S}_{++}^{n}$, our idea is implemented as follows. Let $\mathbb{D}=\left\{\boldsymbol{D}_{1}, \boldsymbol{D}_{2}, \cdots, \boldsymbol{D}_{N}\right\} ; \boldsymbol{D}_{i} \in \mathcal{S}_{++}^{n}$ and $\phi: \mathcal{S}_{++}^{n} \rightarrow \mathcal{H}$ be a Riemannian dictionary and an embedding function on $\mathcal{S}_{++}^{n}$, respectively. Given a Riemannian point $\boldsymbol{X}$, we seek a sparse vector $\boldsymbol{y} \in \mathbb{R}^{N}$ such that $\phi(\boldsymbol{X})$ admits the sparse representation $\boldsymbol{y}$ over $\left\{\phi\left(\boldsymbol{D}_{1}\right), \phi\left(\boldsymbol{D}_{2}\right), \cdots, \phi\left(\boldsymbol{D}_{N}\right)\right\}$. In other words, we are interested in solving the following problem:

$$
l_{\phi}(\boldsymbol{X}, \mathbb{D}) \triangleq \min _{\boldsymbol{y}}\left\|\phi(\boldsymbol{X})-\sum_{j=1}^{N} y_{j} \phi\left(\boldsymbol{D}_{j}\right)\right\|_{2}^{2}+\lambda\|\boldsymbol{y}\|_{1}
$$

For both $J$ and $S$ divergences, an embedding $\phi$ with a reproducing kernel property [49] exists as explained in $\S I I I$. This enables us to use the kernel property $k(\boldsymbol{X}, \boldsymbol{Y})=$ $\phi(\boldsymbol{X})^{T} \phi(\boldsymbol{Y})$ to expand the $\ell_{2}$ term in Eqn. (12) as:

$$
\begin{aligned}
& \left\|\phi(\boldsymbol{X})-\sum_{j=1}^{N} y_{j} \phi\left(\boldsymbol{D}_{j}\right)\right\|_{2}^{2}=\phi(\boldsymbol{X})^{T} \phi(\boldsymbol{X}) \\
- & 2 \sum_{j=1}^{N} y_{j} \phi\left(\boldsymbol{D}_{j}\right)^{T} \phi(\boldsymbol{X})+\sum_{i, j=1}^{N} y_{i} y_{j} \phi\left(\boldsymbol{D}_{i}\right)^{T} \phi\left(\boldsymbol{D}_{j}\right) \\
= & k(\boldsymbol{X}, \boldsymbol{X})-2 \boldsymbol{y}^{T} \mathcal{K}(\boldsymbol{X}, \mathbb{D})+\boldsymbol{y}^{T} \mathbb{K}(\mathbb{D}, \mathbb{D}) \boldsymbol{y},
\end{aligned}
$$

where $\mathcal{K}(\boldsymbol{X}, \mathbb{D})=\left[a_{i}\right]_{N \times 1} ; a_{i}=k\left(\boldsymbol{X}, \boldsymbol{D}_{i}\right)$ and $\mathbb{K}(\mathbb{D}, \mathbb{D})=$ $\left[a_{i j}\right]_{N \times N}$, with $a_{i j}=k\left(\boldsymbol{D}_{i}, \boldsymbol{D}_{j}\right)$. Since $k(\cdot, \cdot)$ is a reproducing kernel, $\mathbb{K}$ is positive definite. This reveals that the optimization problem in Eqn. (13) is convex and similar to its counterpart in Euclidean space, except for the definition of $\mathcal{K}$ and $\mathbb{K}$. Consequently, greedy or relaxation solutions can be adapted to obtain the sparse codes [13]. To solve Eqn. (13) efficiently, we have extended the Feature-Sign Search Algorithm (FSSA) [31] to its kernel version (kFSSA) in Appendix A.

We note that kernel sparse coding and dictionary learning in traditional Euclidean spaces are studied recently in [16], [38]. In contrast, our aim is to obtain sparse coding of points on SPD manifolds, using SPD matrices as dictionary atoms. In our proposed solution this requires dedicated SPD kernels.
Moreover, as will be discussed in $\S \mathrm{V}$ dedicated algorithms for dictionary learning should be devised.

\section{A. Classification Based on Sparse Representation}

If the atoms in the sparse dictionary are not labeled (for example if $\mathbb{D}$ is a generic dictionary not tied to any particular class), the generated sparse codes (vectors) for both training and query data can be fed to Euclidean-based classifiers like support vector machines [5] for classification. In a supervised classification scenario, i.e., if the atoms in sparse dictionary $\mathbb{D}$ are labeled, the generated sparse codes of the query sample can be directly used for classification. Let $\boldsymbol{y}_{i}=\left[y_{0} \delta(l(0)-i), y_{1} \delta(l(1)-i), \cdots, y_{N} \delta(l(N)-i)\right]^{T}$ be the class-specific sparse codes, where $l(j)$ is the class label of atom $\boldsymbol{D}_{j}$ and $\delta(x)$ is the discrete Dirac function [5]. An efficient way of utilizing class-specific dictionary is through computing residual errors [59]. In this case, the residual error of query sample $\boldsymbol{X}$ for class $i$ is defined as:

$$
\varepsilon_{i}(\boldsymbol{X})=\left\|\phi(\boldsymbol{X})-\sum_{j=1}^{N} y_{j} \phi\left(\boldsymbol{D}_{j}\right) \delta(l(j)-i)\right\|^{2} .
$$

Expanding Eqn. (14) and noting that $k(\boldsymbol{X}, \boldsymbol{X})$ is not classdependent, the following expression can be obtained:

$$
\varepsilon_{i}(\boldsymbol{X})=-2 \boldsymbol{y}_{i}^{T} \mathcal{K}(\boldsymbol{X}, \mathbb{D})+\boldsymbol{y}_{i}^{T} \mathbb{K}(\mathbb{D}, \mathbb{D}) \boldsymbol{y}_{i} .
$$

Alternatively, the similarity between query sample $\boldsymbol{X}$ to class $i$ can be defined as $S_{i}(\boldsymbol{X})=h\left(\boldsymbol{y}_{i}\right)$. The function $h(\cdot)$ could be a linear function like $h\left(\boldsymbol{y}_{i}\right)=\boldsymbol{y}_{i}^{T} \mathbf{1}_{N \times 1}$ or even a non-linear one like $h\left(\boldsymbol{y}_{i}\right)=\max \left(\boldsymbol{y}_{i}\right)$. Preliminary experiments suggest that Eqn. (15) leads to higher classification accuracies when compared to the aforementioned alternatives.

\section{B. Computational Complexity}

In terms of computational complexity, we note that the complexity of computing the determinant of an $n \times n$ matrix through Cholesky decomposition is $O\left(\frac{1}{3} n^{3}\right)$. Therefore, computing $S\left(\boldsymbol{X}, \boldsymbol{D}_{i}\right)$ by storing the determinant of dictionary atoms during learning costs $O\left(\frac{2}{3} n^{3}\right)$.

For the $J$ divergence, we note that the inverse of an $n \times n$ SPD matrix can be computed through Cholesky decomposition with $\frac{1}{2} n^{3}$ flops. Therefore, $J\left(\boldsymbol{X}, \boldsymbol{D}_{i}\right)$ can be computed in $2 n^{2.3}+\frac{1}{2} n^{3}$ flops if matrix multiplication is done efficiently. As a result, computing the $J$ divergence is cheaper than computing $S$ divergence for SPD matrices of size less than 35 .

The complexity of sparse coding is dictated by $\mathcal{K}(X, \mathbb{D})$ in Eqn. (15). Neglecting the complexity of the exponential in kernel functions, the complexity of generating Eqn. (15) is $O\left(N\left(2 n^{2.3}+\frac{1}{2} n^{3}\right)\right)$ for $J$ divergence and $O\left(\frac{2 N}{3} n^{3}\right)$ for $S$ divergence.

Note that while the computational complexity is cubic in $n$, it is linear in $N$, i.e., number of dictionary atoms. To give the reader an idea on the speed of the proposed methods, it is worth mentioning that performing sparse coding on $93 \times 93$ covariance descriptors used in $\oint$ VI-A1 took less than 10 and 7 seconds with Jeffrey and Stein divergences, respectively (on an Intel i7 machine using Matlab). Performing a simple nearest neighbor search using AIRM required more than 75 seconds on the same dataset. 


\section{DictionaRY LEARNING}

Given a finite set of observations $\mathbb{X}=\left\{\boldsymbol{X}_{i}\right\}_{i=1}^{m}, \quad \boldsymbol{X}_{i} \in$ $\mathcal{S}_{++}^{n}$, learning a dictionary $\mathbb{D}=\left\{\boldsymbol{D}_{i}\right\}_{i=1}^{N}, \quad \boldsymbol{D}_{i} \in \mathcal{S}_{++}^{n}$ by embedding SPD manifolds into Hilbert space can be formulated as minimizing the following energy function with respect to $\mathbb{D}$ :

$$
f(\mathbb{X}, \mathbb{D}) \triangleq \sum_{i=1}^{m} l_{\phi}\left(\boldsymbol{X}_{i}, \mathbb{D}\right)
$$

Here, $l_{\phi}(\boldsymbol{X}, \mathbb{D})$ is the loss function defined in Eqn. (12). $f(\mathbb{X}, \mathbb{D})$ should be small if $\mathbb{D}$ is "good" at representing the signals $\boldsymbol{X}_{i}$. Among the various solutions to the problem of dictionary learning in Euclidean spaces, iterative methods like K-SVD have received much attention [13]. Borrowing the idea from Euclidean spaces, we propose to minimize the energy in Eqn. (16) iteratively.

To this end, we first initialize the dictionary $\mathbb{D}$ randomly. It is also possible to use intrinsic $k$-means clustering using the Karcher mean [41] to initialize the dictionary. Each iteration of dictionary learning then constitutes of two parts, namely a sparse coding step and a dictionary update step. In the sparse coding step, the dictionary $\mathbb{D}$ is fixed and sparse codes, $\left\{\boldsymbol{y}_{i}\right\}_{i=1}^{m}$ are computed as discussed in $\S$ IV. In the dictionary update step, $\left\{\boldsymbol{y}_{i}\right\}_{i=1}^{m}$ are held fixed while $\mathbb{D}$ is updated, with each dictionary atom updated independently. This resembles the Expectation Maximization (EM) algorithm [12] in nature. In the following subsections, we discuss how dictionary atoms can be updated for both $J$ and $S$ divergences.

\section{A. Dictionary Updates for J Divergence}

As mentioned above, to update $\boldsymbol{D}_{r}$, we keep $\boldsymbol{D}_{j}, j \neq r$ and the sparse codes $\left\{\boldsymbol{y}_{i}\right\}_{i=1}^{m}$ in Eqn. (16) fixed. Generally speaking, one can update $\boldsymbol{D}_{r}$ using gradient descend algorithms on SPD manifolds. This can be done at iteration $t$ by exploiting the tangent space at $\boldsymbol{D}_{r}^{(t)}$ and moving along the direction of steepest descent and utilizing the exponential map to obtain $\boldsymbol{D}_{r}^{(t+1)}$ as a point on $\mathcal{S}_{++}^{n}$.

In this paper, we propose to learn the dictionary in an online manner. Our proposal results in an analytical and closed-form solution for updating dictionary atoms one by one. In contrast to [25], our formulation does not exploit the tangent bundle and exponential maps, and is hence faster and more scalable.

By fixing $\boldsymbol{D}_{j}, j \neq r$ and $\left\{\boldsymbol{y}_{i}\right\}_{i=1}^{m}$, the derivative of Eqn. (16) with respect to $\boldsymbol{D}_{r}$ can be computed as

$$
\begin{aligned}
\frac{\partial f(\mathbb{X}, \mathbb{D})}{\partial \boldsymbol{D}_{r}} & =\sum_{i=1}^{m} \frac{\partial l_{\phi}\left(\boldsymbol{X}_{i}, \mathbb{D}\right)}{\partial \boldsymbol{D}_{r}} \\
& =\sum_{i=1}^{m} \boldsymbol{y}_{i, r}\left(\sum_{j=1}^{N} \boldsymbol{y}_{i, j} \frac{\partial k\left(\boldsymbol{D}_{j}, \boldsymbol{D}_{r}\right)}{\partial \boldsymbol{D}_{r}}-2 \frac{\partial k\left(\boldsymbol{X}_{i}, \boldsymbol{D}_{r}\right)}{\partial \boldsymbol{D}_{r}}\right)
\end{aligned}
$$

For the $J$ divergence, we note that

$$
\nabla_{\boldsymbol{X}} J(\boldsymbol{X}, \boldsymbol{Y})=\frac{1}{2}\left(\boldsymbol{Y}^{-1}-\boldsymbol{X}^{-1} \boldsymbol{Y} \boldsymbol{X}^{-1}\right)
$$

Therefore,

$$
\frac{\partial k_{J}(\boldsymbol{X}, \boldsymbol{Y})}{\partial \boldsymbol{X}}=-\frac{1}{2} \beta k_{J}(\boldsymbol{X}, \boldsymbol{Y})\left(\boldsymbol{Y}^{-1}-\boldsymbol{X}^{-1} \boldsymbol{Y} \boldsymbol{X}^{-1}\right) .
$$

Plugging Eqn. (19) into Eqn. (17) and defining

$$
\begin{aligned}
& \boldsymbol{P}=\sum_{i=1}^{m} \boldsymbol{y}_{i, r}\left(\sum_{j=1}^{N} \boldsymbol{y}_{i, j} k_{J}\left(\boldsymbol{D}_{j}, \boldsymbol{D}_{r}\right) \boldsymbol{D}_{j}^{-1}-2 k_{J}\left(\boldsymbol{X}_{i}, \boldsymbol{D}_{r}\right) \boldsymbol{X}_{i}^{-1}\right), \\
& \boldsymbol{Q}=\sum_{i=1}^{m} \boldsymbol{y}_{i, r}\left(\sum_{j=1}^{N} \boldsymbol{y}_{i, j} k_{J}\left(\boldsymbol{D}_{j}, \boldsymbol{D}_{r}\right) \boldsymbol{D}_{j}-2 k_{J}\left(\boldsymbol{X}_{i}, \boldsymbol{D}_{r}\right) \boldsymbol{X}_{i}\right),
\end{aligned}
$$

then the root of Eqn. (17), i.e., $\partial f(\mathbb{X}, \mathbb{D}) / \partial \boldsymbol{D}_{r}=0$ can be written as:

$$
\boldsymbol{D}_{r}^{-1} \boldsymbol{Q} \boldsymbol{D}_{r}^{-1}=\boldsymbol{P} \text {. }
$$

This equation is identified as a Riccati equation [4]. Its solution is positive definite and given as

$$
\boldsymbol{D}_{r}=\boldsymbol{Q}^{1 / 2}\left(\boldsymbol{Q}^{-1 / 2} \boldsymbol{P}^{-1} \boldsymbol{Q}^{-1 / 2}\right)^{1 / 2} \boldsymbol{Q}^{1 / 2},
$$

provided that both $\boldsymbol{P}$ and $\boldsymbol{Q}$ are positive definite. We note that in deriving the solution, we have assumed that $k_{J}\left(\boldsymbol{D}_{r}, \cdot\right)$ at iteration $t$ can be replaced by $k_{J}\left(\boldsymbol{D}_{r}^{t-1}, \cdot\right)$ and hence $k_{J}\left(\boldsymbol{D}_{r}, \cdot\right)$ are treated as scalars.

\section{B. Dictionary Updates for $S$ Divergence}

Similar to $\S \mathrm{V}$-A, we need to compute the gradient of Eqn. (16) with respect to $\boldsymbol{D}_{r}$, while $\left\{\boldsymbol{y}_{i}\right\}_{i=1}^{m}$ and other atoms are fixed. Noting that

$$
\nabla_{\boldsymbol{X}} S(\boldsymbol{X}, \boldsymbol{Y})=(\boldsymbol{X}+\boldsymbol{Y})^{-1}-\frac{1}{2} \boldsymbol{X}^{-1},
$$

the solution of $\partial f(\mathbb{X}, \mathbb{D}) / \partial \boldsymbol{D}_{r}=0$ with $k_{S}(\cdot, \cdot)$ can be written as:

$$
\begin{aligned}
& \sum_{i=1}^{m} \boldsymbol{y}_{i, r}\left(2 k_{S}\left(\boldsymbol{X}_{i}, \boldsymbol{D}_{r}\right)\left(\left(\boldsymbol{X}_{i}+\boldsymbol{D}_{r}\right)^{-1}-\frac{1}{2} \boldsymbol{D}_{r}^{-1}\right)\right)= \\
& \sum_{i=1}^{m} \boldsymbol{y}_{i, r}\left(\sum_{j=1}^{N} \boldsymbol{y}_{i, j} k_{S}\left(\boldsymbol{D}_{j}, \boldsymbol{D}_{r}\right)\left(\left(\boldsymbol{D}_{j}+\boldsymbol{D}_{r}\right)^{-1}-\frac{1}{2} \boldsymbol{D}_{r}^{-1}\right)\right)
\end{aligned}
$$

Since Eqn. (24) contains inverses and kernel values, a closedform solution for computing $\boldsymbol{D}_{r}$ cannot be sought. As such, we propose an alternative solution by exploiting previous values of $\left(\boldsymbol{D}_{i}+\boldsymbol{D}_{r}\right)^{-1}$ in the update step. More specifically, rearranging Eqn. (24) and replacing $k\left(\cdot, \boldsymbol{D}_{r}\right)$ as well as $\left(\boldsymbol{D}_{i}+\boldsymbol{D}_{r}\right)^{-1}$ by their previous values, atom $\boldsymbol{D}_{r}$ at iteration $t+1$ is updated according to:

$$
\boldsymbol{D}_{r}^{(t+1)}=\frac{2 \boldsymbol{P}^{-1}}{\sum_{i=1}^{m} \boldsymbol{y}_{i, r}\left(2 k_{S}\left(\boldsymbol{X}_{i}, \boldsymbol{D}_{r}\right)-\sum_{j=1}^{N} \boldsymbol{y}_{i, j} k_{S}\left(\boldsymbol{D}_{j}, \boldsymbol{D}_{r}\right)\right)},
$$

where

$$
\begin{aligned}
\boldsymbol{P} & =\sum_{i=1}^{m} \boldsymbol{y}_{i, r}\left(2 k_{S}\left(\boldsymbol{X}_{i}, \boldsymbol{D}_{r}\right)\left(\boldsymbol{X}_{i}+\boldsymbol{D}_{r}^{(t)}\right)^{-1}\right. \\
& \left.-\sum_{j=1}^{N} \boldsymbol{y}_{i, j} k_{S}\left(\boldsymbol{D}_{j}, \boldsymbol{D}_{r}\right)\left(\boldsymbol{D}_{j}+\boldsymbol{D}_{r}^{(t)}\right)^{-1}\right) .
\end{aligned}
$$

\section{Practical Considerations}

The dictionary update in Eqn. (22) results in an SPD matrix provided that matrices $\boldsymbol{P}$ and $\boldsymbol{Q}$ are SPD. In practice, this might not be the case and as such projection to the positive definite cone is required. The same argument holds for Eqn. (25). Given an arbitrary square matrix $\boldsymbol{A} \in \mathbb{R}^{n \times n}$, the problem of finding the closest SPD matrix to $\boldsymbol{A}$ has 
received considerable attention in the literature (c.f., [24]). While projecting onto positive definite cone can be achieved by thresholding (i.e., replacing negative eigenvalues by a small positive number), a more principal approach can be used as follows. If square matrix $\boldsymbol{X}$ is positive definite then $\boldsymbol{X}+\boldsymbol{X}^{T}$ is also positive definite. As such, the following convex problem can be solved ${ }^{3}$ to obtain the closest SPD matrix $\boldsymbol{X}$ to the square matrix $\boldsymbol{A}$ :

$$
\min \|\boldsymbol{A}-\boldsymbol{X}\|_{F} \quad \text { s.t. } \boldsymbol{X}+\boldsymbol{X}^{T} \succ 0
$$

We note that the formulation provided here works for nonsymmetric matrix $\boldsymbol{A}$ as well. This is again useful in practice as numerical issues might create non-symmetric matrices (e.g., $\boldsymbol{P}$ and $\boldsymbol{Q}$ in Eqn. (22) might not become symmetric due to the limited numerical accuracy in a computational implementation).

\section{EXPERIMENTS}

Two sets of experiments ${ }^{4}$ are presented in this section. In the first set, we evaluate the performance of the proposed sparse coding methods (as described in $\S$ IV) without dictionary learning. This is to contrast sparse coding to previous stateof-the-art methods on several popular closed-set classification tasks. To this end, each point in the training set is considered as an atom in the dictionary. Since the atoms in the dictionary are labeled in this case, the residual error approach for classification (as described in $\S$ IV-A) will be used to determine the label of a query point. In the second set of experiments, the performance of the sparse coding methods is evaluated in conjunction with the proposed dictionary learning algorithms described in $\S$ V. For brevity, we denote Riemannian sparse representation with $J$ divergence as RSR-J, and the $S$ divergence counterpart as RSR-S.

The first priority of the experiments is to contrast the proposed methods against recent techniques designed to work on SPD manifolds. That is, the tasks and consequently the datasets were chosen to enable fair comparisons against stateof-the-art SPD methods. While exploring other visual tasks such as face verification [8] is beyond the scope of this paper, it is an interesting path to pursue in future work.

\section{A. Sparse Coding}

Below, we compare and contrast the performance of RSR-J and RSR-S methods against state-of-the-art techniques in five classification tasks, namely action recognition from $3 \mathrm{D}$ skeleton data, face recognition, material classification, person re-identification and texture categorization.

1) Action Recognition from 3D Skeleton Sequences: We used the motion capture HDM05 dataset [37] for the task of action recognition from skeleton data. Each action is encoded by the locations of 31 joints over time, with the speed of 120 frames per second. Given an action by $K$ joints over $m$ frames, we extracted the joint covariance descriptor [26] which is an SPD matrix of size $3 K \times 3 K$ as follows. Let $x_{i}(t), y_{i}(t)$ and

\footnotetext{
${ }^{3} \mathrm{~A}$ solver like CVX [17] can be used.

${ }^{4}$ The corresponding Matlab/Octave source code is available at http://nicta.com.au/people/mharandi
}

TABLE I: Recognition accuracy (in \%) for the HDM05-MOCAP dataset [37]

\begin{tabular}{lc}
\hline Method & Recognition Accuracy \\
\hline logEuc-SC [18] & $89.9 \%$ \\
Cov3DJ [26] & $95.4 \%$ \\
\hline RSR-J & $\mathbf{9 8 . 2} \%$ \\
RSR-S & $97.3 \%$ \\
\hline
\end{tabular}

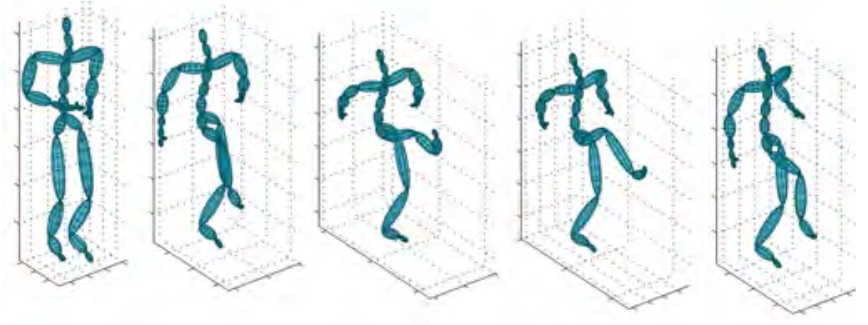

Fig. 1: Example of a kicking action from the HDM05 action dataset [37].

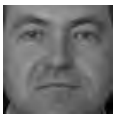

(a) ba

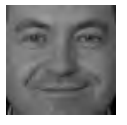

(b) bj

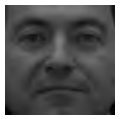

(c) bk

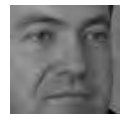

(d) bd

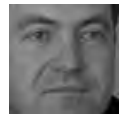

(e) be

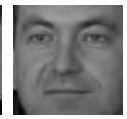

(f) bf

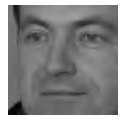

(g) bg
Fig. 2: Examples from the FERET face dataset [42]

$z_{i}(t)$ be the $x, y$, and $z$ coordinates of the $i$-th joint at frame $t$. Let $\boldsymbol{f}(t)$ be the vector of all joint locations at time $t$, i.e., $\boldsymbol{f}(t)=\left[x_{1}(t), \cdots, x_{K}(t), y_{1}(t), \cdots, y_{K}(t), z_{1}(t), \cdots, z_{K}(t)\right]^{T}$, which has $3 K$ elements. The action represented over $m$ frames is then described by the covariance of vectors $\boldsymbol{f}(t)$.

We used 3 subjects (140 action instances) for training, and the remaining 2 subjects (109 action instances) for testing. The set of actions used in this experiment is: "clap above head', 'deposit floor', 'elbow to knee', 'grab high', 'hop both legs', 'jog', 'kick forward', 'lie down floor', 'rotate both arms backward', 'sit down chair', 'sneak', 'squat', 'stand up lie' and 'throw basketball'.

In Table I we compare the performance of RSR-J and RSR-S against logEuc-SC [18] and Cov3DJ [26]. The TSC algorithm [51] does not scale well to large SPD matrices and thus is not considered here. Cov3DJ encodes the relationship between joint movement and time by deploying multiple covariance matrices over sub-sequences in a hierarchical fashion. The results show that in this case RSR-J is better than RSR-S. Furthermore, both RSR-J and RSR-S outperform logEuc-SC and Cov3DJ.

2) Face Recognition: We used the 'b' subset of the FERET dataset [42], which includes 1800 images from 200 subjects. The images were closely cropped around the face and downsampled to $64 \times 64$. Examples are shown in Figure 2 .

We performed four tests with various pose angles. Training data was composed of images marked 'ba', 'bj' and 'bk' (i.e., frontal faces with expression and illumination variations). Images with 'bd', 'be', 'bf', and 'bg' labels (i.e., non-frontal faces) were used as test data. 
TABLE II: Recognition accuracy (in \%) for the FERET face dataset [42].

\begin{tabular}{lccccc}
\hline Method & bd & be & bf & bg & average \\
\hline SRC [59] & $27.5 \%$ & $55.5 \%$ & $61.0 \%$ & $26.0 \%$ & $42.5 \%$ \\
GSRC [61] & $77.0 \%$ & $93.5 \%$ & $97.0 \%$ & $79.0 \%$ & $86.6 \%$ \\
\hline logEuc-SC [18] & $74.0 \%$ & $94.0 \%$ & $97.5 \%$ & $80.5 \%$ & $86.5 \%$ \\
TSC [51] & $36.0 \%$ & $73.0 \%$ & $73.5 \%$ & $44.5 \%$ & $56.8 \%$ \\
\hline RSR-J & $\mathbf{8 2 . 5} \%$ & $94.5 \%$ & $\mathbf{9 8 . 0} \%$ & $83.5 \%$ & $89.6 \%$ \\
RSR-S & $79.5 \%$ & $\mathbf{9 6 . 5} \%$ & $97.5 \%$ & $\mathbf{8 6 . 0} \%$ & $\mathbf{8 9 . 9} \%$ \\
\hline
\end{tabular}

Each face image is described by a $43 \times 43$ SPD matrix using the following features:

$$
\boldsymbol{f}_{x, y}=\left[I(x, y), x, y,\left|G_{0,0}(x, y)\right|, \cdots,\left|G_{4,7}(x, y)\right|\right]^{T},
$$

where $I(x, y)$ is the intensity value at position $(x, y),|\cdot|$ denotes the magnitude of a complex value and $G_{u, v}(x, y)$ is the response of a 2D Gabor wavelet centered at $(x, y)$ with orientation $u$ and scale $v$. In this work, we followed [40] and generated 40 Gabor filters in 8 orientations and 5 scales.

The proposed methods are compared against TSC [51], logEuc-SC [18], Sparse Representation-based Classification (SRC) [59] and its Gabor-based extension (GSRC) [61]. For SRC, PCA was used to reduce the dimensionality of data. We evaluated the performance of SRC for various dimensions of PCA space and the maximum performance is reported. For the GSRC algorithm [61], we followed the recommendations of the authors for the downsampling factor in Gabor filtering. As for the $\operatorname{logEuc}-\mathrm{SC}$, we consider a kernel extension of the original algorithm. In other words, instead of directly using $\log (\cdot)$ representations in a sparse coding framework as done in [18], we consider a kernel extension on log representations using an RBF kernel. The kernel extension of sparse coding is discussed in depth in [16], [38]. This enhances the results in all cases and makes the logEuc-SC and RSR methods more comparable.

Table II shows the performance of all the studied methods for the task of face recognition. Both RSR-J and RSR-S outperform other methods, with RSR-S being marginally better than RSR-J.

3) Material Categorization: We used the Flickr dataset [48] for the task of material categorization. The dataset contains ten categories of materials: fabric, foliage, glass, leather, metal, paper, plastic, stone, water and wood. Each category has 100 images, 50 of which are close-up views and the remaining 50 are views at object-scale (see Figure 3 for examples). A binary, human-labeled mask is provided for each image in the dataset, describing the location of the object in the image. We only consider pixels inside this binary mask for material recognition and disregard all background pixels. SIFT [35] features have recently been shown to be robust and discriminative for material classification [33]. We therefore constructed RCMs of size $155 \times 155$ using 128 dimensional SIFT features (only from gray-scaled images) and 27 dimensional color descriptors. To this end, SIFT descriptors were computed at points on a regular grid with 5 pixel spacing. The color descriptor was obtained by simply stacking colors from $3 \times 3$ patches centered at grid points.

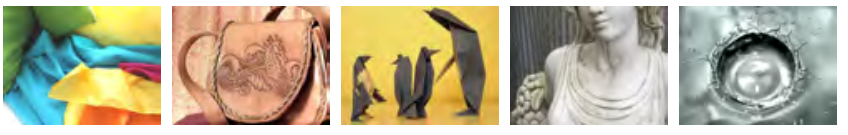

Fig. 3: Examples from the Flickr dataset [48].

TABLE III: Recognition accuracy (in \%) along its standard deviation for the Flickr dataset [48].

\begin{tabular}{lc}
\hline Method & Recognition Acc. \\
\hline VZ [56] & $23.8 \% \pm N / A$ \\
VZ-augmented [34] & $37.4 \% \pm N / A$ \\
SD [33] & $29.9 \% \pm N / A$ \\
aLDA [34] & $44.6 \% \pm N / A$ \\
RSR-J & $44.0 \% \pm 3.0$ \\
RSR-S & $\mathbf{5 1 . 4} \% \pm \mathbf{1 . 9}$ \\
\hline
\end{tabular}

Table III compares the performance of the proposed methods against the state-of-the-art non-parametric geometric detail extraction method (SD) [33], augmented Latent Dirichlet Allocation (aLDA) [34], and the texton-based representation introduced in [56]. The results indicate that RSR-S considerably outperforms previous state-of-the-art approaches. We also note that RSR-J outperforms methods proposed in [33], [56] by a large margin and is only slightly worse than the aLDA algorithm [34].

4) Person Re-identification: We used the modified ETHZ dataset [47]. The original ETHZ dataset was captured using a moving camera [14], providing a range of variations in the appearance of people. The dataset is structured into 3 sequences. Sequence 1 contains 83 pedestrians (4,857 images), Sequence 2 contains 35 pedestrians (1,936 images), and Sequence 3 contains 28 pedestrians (1,762 images). See left panel of Fig. 4 for examples.

We downsampled all images to $64 \times 32$ pixels. For each subject we randomly selected 10 images for training and used the rest for testing. Random selection of training and testing data was repeated 20 times to obtain reliable statistics. To describe each image, the covariance descriptor was computed using the following features:

$$
\boldsymbol{f}_{\boldsymbol{u}}=\left[\boldsymbol{u}, R_{\boldsymbol{u}}, G_{\boldsymbol{u}}, B_{\boldsymbol{u}}, \dot{R}_{\boldsymbol{u}}, \dot{G}_{\boldsymbol{u}}, \dot{B}_{\boldsymbol{u}}, \ddot{R}_{\boldsymbol{u}}, \ddot{G}_{\boldsymbol{u}}, \ddot{B}_{\boldsymbol{u}}\right]^{T}
$$

where $\boldsymbol{u}=[x, y]$ is the position of a pixel, while $R_{\boldsymbol{u}}, G_{\boldsymbol{u}}$ and $B_{\boldsymbol{u}}$ represent the corresponding color information. The gradient and Laplacian for color $C$ are represented by $\dot{C}_{\boldsymbol{u}}=$ $[|\partial C / \partial x|,|\partial C / \partial y|]$ and $\ddot{C}_{\boldsymbol{u}}=\left[\left|\partial^{2} C / \partial x^{2}\right|,\left|\partial^{2} C / \partial y^{2}\right|\right]$, respectively.

We compared the proposed RSR methods with several techniques previously used for pedestrian detection: SymmetryDriven Accumulation of Local Features (SDALF) [2], Riemannian Locality Preserving Projection (RLPP) [22], and log-Euclidean sparse coding [18]. The results for TSC [51] could not be generated in a timely manner due to the heavy computational load of the algorithm.

Results for the first two sequences are shown in Fig. 4, in terms of cumulative matching characteristic (CMC) curves. The CMC curve represents the expectation of finding the correct match in the top $n$ matches. The proposed RSR-S method obtains the highest accuracy on both sequences. RSR-J 
outperforms SDALF, RLPP and log-Euclidean sparse coding on sequence one. For the second sequence, RLPP and SDALF perform better than RSR-J for low ranks while RSR-J outperforms them for rank higher than two.

For Sequence 3 (not shown), very similar performances are obtained by SDALF, RLPP and the proposed methods. For this sequence, RSR-J and RSR-S achieve rank 1 accuracy of $98.3 \%$ and $98.7 \%$, respectively. The CMC curves are almost saturated at perfect recognition at rank 3 for both RSR-J and RSR-S methods.

5) Texture Classification: We performed a classification task using the Brodatz texture dataset [43]. Examples are shown in Fig. 5. We followed the test protocol devised in [51] and generated nine test scenarios with various number of classes. This includes 5-texture ('5c', '5m', '5v', '5v2', '5v3'), 10-texture ('10', '10v') and 16-texture ('16c', '16v') mosaics. To create a Riemannian manifold, each image was first downsampled to $256 \times 256$ and then split into 64 regions of size $32 \times 32$. The feature vector for any pixel $I(x, y)$ is $\boldsymbol{f}(x, y)=$ $\left[I(x, y),|\partial I / \partial x|,|\partial I / \partial y|,\left|\partial^{2} I / \partial x^{2}\right|,\left|\partial^{2} I / \partial y^{2}\right|\right]^{T}$. Each region is described by a $5 \times 5$ covariance descriptor of these features. For each test scenario, five covariance matrices per class were randomly selected as training data and the rest was used for testing. The random selection of training/testing data was repeated 20 times.

Fig. 6 compares the proposed RSR methods against logEucSC [18] and TSC [51]. In general, the proposed methods obtain the highest recognition accuracy on all test scenarios except for the ' $5 \mathrm{c}$ ' test, where both methods have slightly worse performance than TSC. We note that in some cases such as '5m' and '5v2', RSR-J performs better than RSR-S. However, RSR-S is overall a slightly superior method for this task.

\section{B. Dictionary Learning}

Here we analyze the performance of the proposed dictionary learning techniques as described in $\S \mathrm{V}$ on two classification tasks: texture classification and action recognition.

1) Texture Classification: Here we consider a multi-class classification problem, using 111 texture images of the Brodatz texture dataset [43]. From each image we randomly extracted 50 blocks of size $32 \times 32$. To train the dictionary, 20 blocks from each image were randomly selected, resulting in a dictionary learning problem with 2200 samples. From the remaining blocks, 20 per image were used as probe data and 10 as gallery samples. The process of random block creation and dictionary generation was repeated twenty times. The average recognition accuracies over probe data are reported here. In the same manner as in $\S$ VI-A5, we used the feature vector $\boldsymbol{f}(x, y)=$ $\left[I(x, y),|\partial I / \partial x|,|\partial I / \partial y|,\left|\partial^{2} I / \partial x^{2}\right|,\left|\partial^{2} I / \partial y^{2}\right|\right]^{T}$ to create the covariance, where the first dimension is the grayscale intensity, and the remaining dimensions capture first and second order gradients.

We used the proposed methods to obtain the sparse codes, coupled with a dictionary generated via two separate methods: intrinsic $k$-means, and the proposed learning algorithm $(\S \mathrm{V})$. The sparse codes were then classified using a nearest-neighbor classifier.
Figure 7 shows the performance of RSR-J and RSR-S for various dictionary sizes. Red curves show the performance when the intrinsic $k$-means algorithm was utilized for dictionary learning. The blue curves demonstrate the recognition accuracies when the methods proposed in $\S \mathrm{V}$ were used for training, and finally the green curves show the performance of log-Euclidean sparse coding equipped with KSVD [1] algorithm for dictionary learning. The figures show that the proposed dictionary learning approach consistently outperforms $k$-means bar one case (RSR-J for dictionary size 8). Using the proposed dictionary learning approach, RSR-J achieves the maximum recognition accuracy of $63.3 \%$ with 104 atoms, while RSR-S obtains the maximum accuracy of $60.6 \%$ with 24 atoms. In contrast, when intrinsic $k$-means is used for dictionary learning, the maximum recognition accuracies for RSR-J and RSR-S are $52.9 \%$ and $53.2 \%$, respectively. Furthermore, in all cases RSR-S is superior to the log-Euclidean solution, while RSR-J performs better than the log-Euclidean approach only for dictionaries with size larger than 24 atoms.

2) Action Recognition: The UCF sport action dataset [44] consists of ten categories of human actions including swinging on the pommel horse, driving, kicking, lifting weights, running, skateboarding, swinging at the high bar, swinging golf clubs, and walking (examples of a diving action are shown in Fig. 8). The number of videos for each action varies from 6 to 22 and there are 150 video sequences in total. Furthermore, the videos presented in this dataset have non-uniform backgrounds and both the camera and the subject are moving in some actions. Frames in all video sequences are cropped according to the region of interest provided with the dataset and then resized to $64 \times 64$. The standard protocol in this dataset is the leave-one-out (LOO) cross validation [28], [44], [60].

From each video, we extracted several RCMs by splitting video data into $3 \mathrm{D}$ volumes. Volumes had the size of $32 \times 32 \times 15$ in $x-y-t$ domains with 8 pixels shift in each direction. From each volume, a $12 \times 12 \mathrm{RCM}$ was extracted using kinematic features described in [18]. From training RCMs, we learned separate dictionaries for $J$ and $S$ divergences using the methods described in $\S \mathrm{V}$ with 256 atoms each. The dictionaries were then used to determine the video descriptor. To this end, each video was described by simply pooling the sparse codes of its $32 \times 32 \times 15$ volumes using max operator. Having training and testing descriptors at our disposal, a linear SVM [5] was used as classifier.

In Table IV, the overall performance of the RSR-J and RSR-S methods is compared against three state-of-the-art Euclidean approaches: HOG3D [57], Hierarchy of Discriminative space-time Neighbourhood features (HDN) [28], and augmented features [60] in conjunction with multiple kernel learning (AFMKL). HOG3D is an extension of histogram of oriented gradient descriptor [11] to spatio-temporal spaces. HDN learns shapes of space-time feature neighbourhoods that are most discriminative for a given action category. The idea is to form new features composed of the neighbourhoods around the interest points in a video. AFMKL exploits appearance distribution features and spatio-temporal context features in a learning scheme for action recognition. As shown in Ta- 


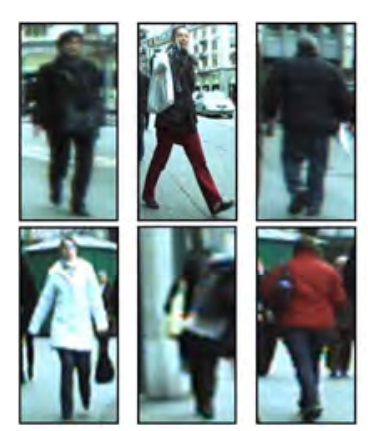

(a) Sample images

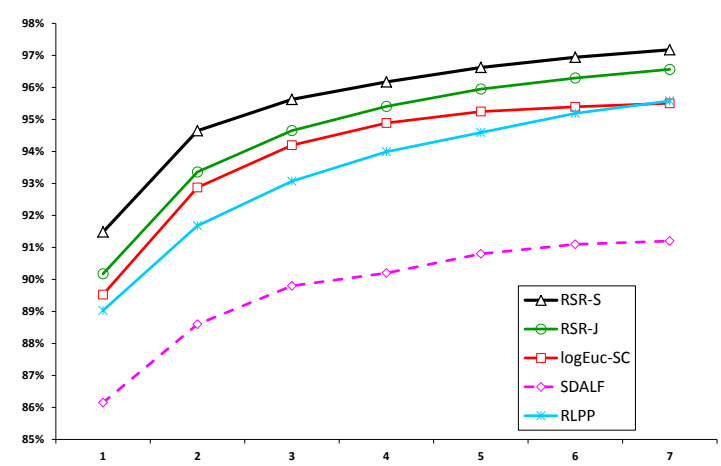

(b) Seq. \#1

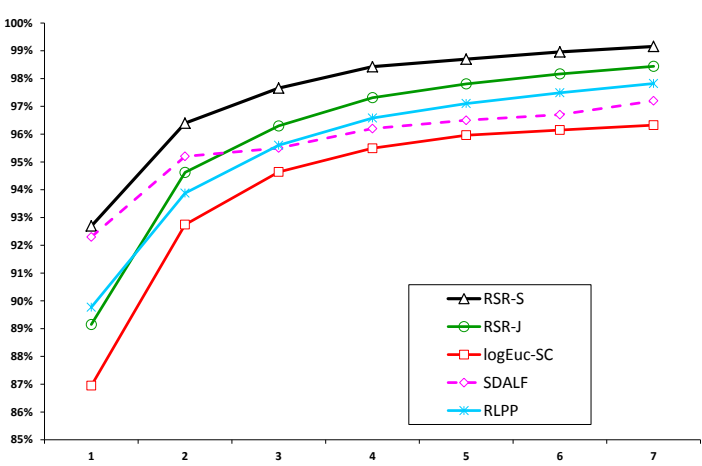

(c) Seq. \#2

Fig. 4: Person Re-identification using the ETHZ dataset [14]. Left column, examples of pedestrians in the ETHZ dataset. Middle column, results on Seq. \#1; right column, on Seq. \#2. The proposed RSR-J and RSR-S methods are compared with SDALF [2], RLPP [22] and log-Euclidean sparse coding (logEuc-SC) [18].

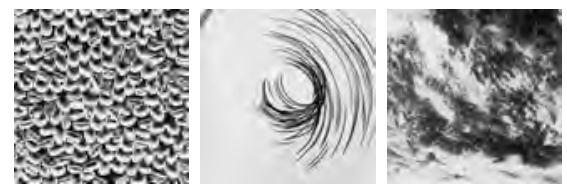

Fig. 5: Examples from the Brodatz texture dataset [43].

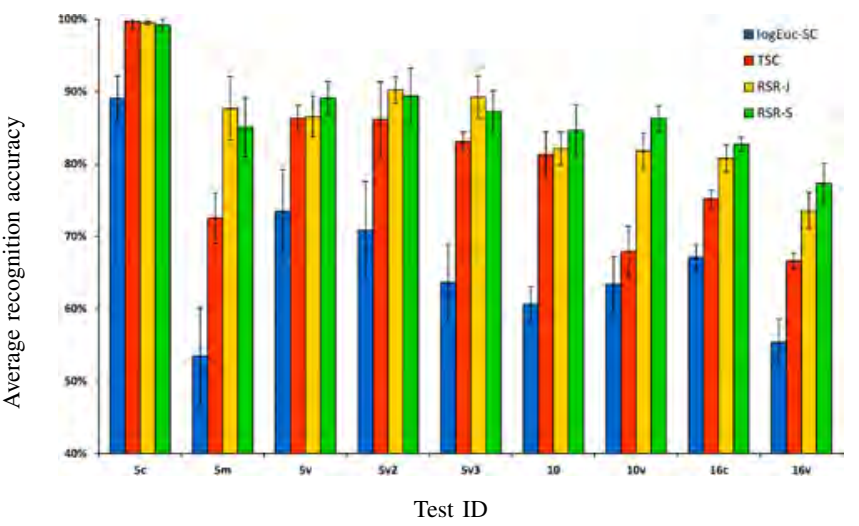

Fig. 6: Average recognition accuracy on the Brodatz texture dataset [43] using log-Euclidean sparse representation (logEucSC) [18], Tensor Sparse Coding (TSC) [51] and the proposed RSR-J and RSR-S approaches. The black bars indicate standard deviations.

TABLE IV: Recognition accuracy (in \%) for the UCF action recognition dataset using HOG3D, HDN [28], AFMKL [60] and the proposed RSR-J and RSR-S approaches.

\begin{tabular}{lc}
\hline Method & Recognition Accuracy \\
\hline HOG3D [57] & 85.6 \\
HDN [28] & 87.3 \\
AFMKL [60] & 91.3 \\
logEuc-SC with dic. learning & 89.3 \\
RSR-J & 90.7 \\
RSR-S & $\mathbf{9 4 . 0}$ \\
\hline
\end{tabular}

ble IV, RSR-J outperforms the log-Euclidean approach and is marginally worse than AMFKL. RSR-S achieves the highest overall accuracy.

The confusion matrices for RSR-J and RSR-S divergences are shown in Tables V and VI, respectively. RSR-J perfectly classifies the actions of diving, golf swinging, kicking, riding horse, high bar swinging, walking and lifting, while RSR-S achieves perfect classification on golf swinging, riding horse, running, high bar swinging and lifting. Nevertheless, the overall performance of RSR-S surpasses that of RSR-J since RSR-J performs poorly in classifying the pommel-horse action.

\section{MAIN FINDINGS AND FUTURE WORK}

With the aim of addressing sparse representation on SPD manifolds, we proposed to seek the solution through embedding the manifolds into RKHS with the aid of two Bregman divergences, namely Stein and Jeffrey divergences. This led to a relaxed and extended version of the Lasso problem [13] on SPD manifolds.

In Euclidean spaces, the success of many learning algorithms arises from their use of kernel methods [49]. Therefore, one could expect embedding a Riemannian manifold into higher dimensional Reproducing Kernel Hilbert Space (RKHS), where linear geometry applies, facilitates inference. Such an embedding, however, requires a non-trivial kernel function defined on the manifold, which, according to Mercer's theorem [49], must be positive definite. The approach introduced here attains its merit from the following facts:

- By recasting the sparse coding from $\mathcal{S}_{++}^{n}$ into RKHS, a convex problem is obtained which can be solved quite efficiently. The sparse coding problem is in effect linearized, which is far easier than solving the Riemannian version of sparse coding as depicted in Eqn. (11).

- Recasting the sparse coding from $\mathcal{S}_{++}^{n}$ into RKHS exploits the advantages of higher dimensional Hilbert spaces, such as easier separability of classes.

- The $J$ and $S$ divergences used in this paper are closely related to the Affine Invariant Riemannian Metric (AIRM) [41], and have several useful properties such as invariance to inversion and affine transforms. However, unlike AIRM, the $J$ and $S$ divergences admit a Hilbert space embedding (i.e., can be converted to kernel functions).

Experiments on several classification tasks show that the proposed approaches achieve notable improvements in dis- 

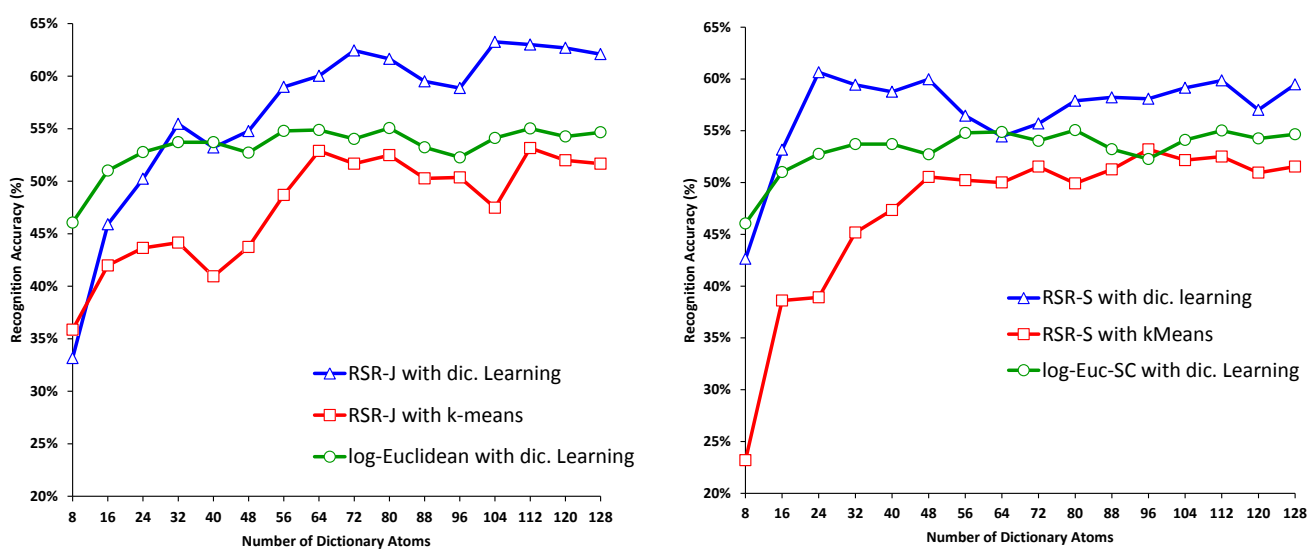

Fig. 7: Comparison of recognition accuracy versus size of dictionary for RSR-J and RSR-S. The red curve shows the accuracy for dictionaries learned by intrinsic $k$-means algorithm. The green curve shows the accuracy for dictionaries learned by log-Euclidean method, that is dictionary learning (K-SVD) along sparse coding on the identity tangent space. The blue curve shows the accuracy for the proposed learning approach.

TABLE V: Confusion matrix (in \%) for the RSR-J method on the UCF sport action dataset using LOO protocol.

\begin{tabular}{lllllllllll}
\hline & D & GS & K & RH & R & S & PH & HS & W & L \\
\hline D & $\mathbf{1 0 0}$ & 0 & 0 & 0 & 0 & 0 & 0 & 0 & 0 & 0 \\
GS & 0 & $\mathbf{1 0 0}$ & 0 & 0 & 0 & 0 & 0 & 0 & 0 & 0 \\
K & 0 & 0 & $\mathbf{1 0 0}$ & 0 & 0 & 0 & 0 & 0 & 0 & 0 \\
RH & 0 & 0 & 0 & $\mathbf{1 0 0}$ & 0 & 0 & 0 & 0 & 0 & 0 \\
R & 0 & 0 & 0 & 0 & $\mathbf{9 1 . 7}$ & 0 & 0 & 0 & 0 & 8.3 \\
S & 0 & 0 & 23.1 & 0 & 0 & $\mathbf{6 9 . 2}$ & 0 & 7.7 & 0 & 0 \\
PH & 0 & 16.65 & 16.65 & 0 & 0 & 0 & $\mathbf{2 5 . 0}$ & 0 & 0 & 41.7 \\
HS & 0 & 0 & 0 & 0 & 0 & 0 & 0 & $\mathbf{1 0 0}$ & 0 & 0 \\
W & 0 & 0 & 0 & 0 & 0 & 0 & 0 & 0 & $\mathbf{1 0 0}$ & 0 \\
L & 0 & 0 & 0 & 0 & 0 & 0 & 0 & 0 & 0 & $\mathbf{1 0 0}$ \\
\hline
\end{tabular}
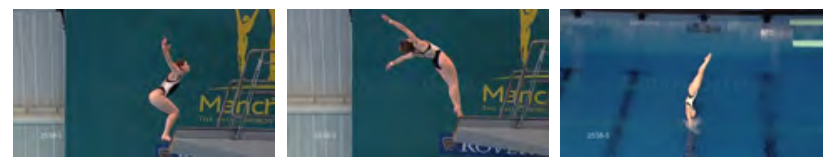

Fig. 8: Examples from the UCF sport action dataset [44].

crimination accuracy, in comparison to state-of-the-art methods such as tensor sparse coding [51]. We conjuncture that this stems from better exploitation of Riemannian geometry, as both divergences enjoy several properties similar to affine invariant Riemannian metric on SPD manifolds.

We have furthermore proposed algorithms for learning a dictionary, closely tied to the Stein and Jeffrey divergences. The experiments show that in many cases better performance is achieved with RSR-S as compared to RSR-J. However, we note that Jeffrey divergence enjoys several unique properties (e.g., closed form solution for averaging and Hilbert space embedding for all values of $\beta$ in Eqn. (6)) which makes it attractive for analyzing SPD matrices. Future venues of exploration include devising other types of inference and machineries based on $J$ and $S$ divergences, such as structured learning.

\section{APPENDIX}

Geometric Mean of J-Divergence

Theorem 1. For two matrices $\boldsymbol{A}, \boldsymbol{B} \in \mathcal{S}_{++}^{n}$, the geometric mean of $J$ divergence $\boldsymbol{A} \sharp_{J} \boldsymbol{B}$ and AIRM $\boldsymbol{A} \sharp_{R} \boldsymbol{B}$ are the same.
TABLE VI: Confusion matrix (in \%) for the RSR-S method on the UCF sport action dataset using LOO protocol.

\begin{tabular}{lllllllllll}
\hline & $\mathbf{D}$ & $\mathbf{G S}$ & $\mathbf{K}$ & $\mathbf{R H}$ & $\mathbf{R}$ & $\mathbf{S}$ & $\mathbf{P H}$ & $\mathbf{H S}$ & $\mathbf{W}$ & $\mathbf{L}$ \\
\hline $\mathbf{D}$ & $\mathbf{9 2 . 9}$ & 0 & 0 & 0 & 0 & 0 & 0 & 7.1 & 0 & 0 \\
$\mathbf{G S}$ & 0 & $\mathbf{1 0 0}$ & 0 & 0 & 0 & 0 & 0 & 0 & 0 & 0 \\
$\mathbf{K}$ & 0 & 0 & $\mathbf{9 5 . 0}$ & 0 & 15 & 0 & 0 & 5.0 & 0 & 0 \\
$\mathbf{R H}$ & 0 & 0 & 0 & $\mathbf{1 0 0}$ & 0 & 0 & 0 & 0 & 0 & 0 \\
$\mathbf{R}$ & 0 & 0 & 0 & 0 & $\mathbf{1 0 0}$ & 0 & 0 & 0 & 0 & 0 \\
$\mathbf{S}$ & 7.7 & 0 & 15.4 & 0 & 0 & $\mathbf{6 9 . 2}$ & 7.7 & 0 & 0 & 0 \\
$\mathbf{P}$ & 0 & 0 & 0 & 0 & 0 & 0 & $\mathbf{8 3 . 3}$ & 0 & 0 & 16.7 \\
$\mathbf{H}$ & 0 & 0 & 0 & 0 & 0 & 0 & 0 & $\mathbf{1 0 0}$ & 0 & 0 \\
$\mathbf{W}$ & 7.7 & 0 & 0 & 0 & 0 & 0 & 0 & 0 & $\mathbf{9 2 . 3}$ & 0 \\
$\mathbf{L}$ & 0 & 0 & 0 & 0 & 0 & 0 & 0 & 0 & 0 & $\mathbf{1 0 0}$ \\
\hline
\end{tabular}

Proof. For the $J$ divergence, we note that

$\frac{\partial\{J(\boldsymbol{X}, \boldsymbol{A})+J(\boldsymbol{X}, \boldsymbol{B})\}}{\partial \boldsymbol{X}}=\frac{1}{2}\left(\boldsymbol{A}^{-1}+\boldsymbol{B}^{-1}-\boldsymbol{X}^{-1}(\boldsymbol{A}+\boldsymbol{B}) \boldsymbol{X}^{-1}\right)$.

Therefore, $\boldsymbol{A}_{J} \boldsymbol{B}$ is the solution of

$$
\boldsymbol{X}\left(\boldsymbol{A}^{-1}+\boldsymbol{B}^{-1}\right) \boldsymbol{X}=\boldsymbol{A}+\boldsymbol{B},
$$

which is a Riccati equation with only one positive definite solution [4]. We note that

$$
\begin{aligned}
\boldsymbol{A}_{\sharp_{R}} \boldsymbol{B} & =\exp _{\boldsymbol{B}}\left(\frac{1}{2} \log _{\boldsymbol{B}}(\boldsymbol{A})\right)=\exp _{\boldsymbol{A}}\left(\frac{1}{2} \log _{\boldsymbol{A}}(\boldsymbol{B})\right) \\
& =\boldsymbol{A}^{\frac{1}{2}}\left(\boldsymbol{A}^{-\frac{1}{2}} \boldsymbol{B} \boldsymbol{A}^{-\frac{1}{2}}\right)^{\frac{1}{2}} \boldsymbol{A}^{\frac{1}{2}} .
\end{aligned}
$$

It can be readily shown that $\boldsymbol{A} \sharp_{R} \boldsymbol{B}$ satisfies Eqn. (28) which concludes the proof.

\section{Kernelized Feature Sign Algorithm}

The efficiency of the Feature-Sign Search algorithm [31] for finding sparse codes in vector spaces has been analyzed in [30]. The algorithm was shown to outperform (in terms of speed and accuracy) sparse solvers such as the generic QP solver [17]. The gain is even higher for large and highdimensional datasets (which are common in computer vision tasks). As such, we have elected to recast the algorithm into its RKHS version, in order to find the sparse codes on SPD manifolds. We summarize the new version below, with the pseudo-code shown in Algorithm 1. 


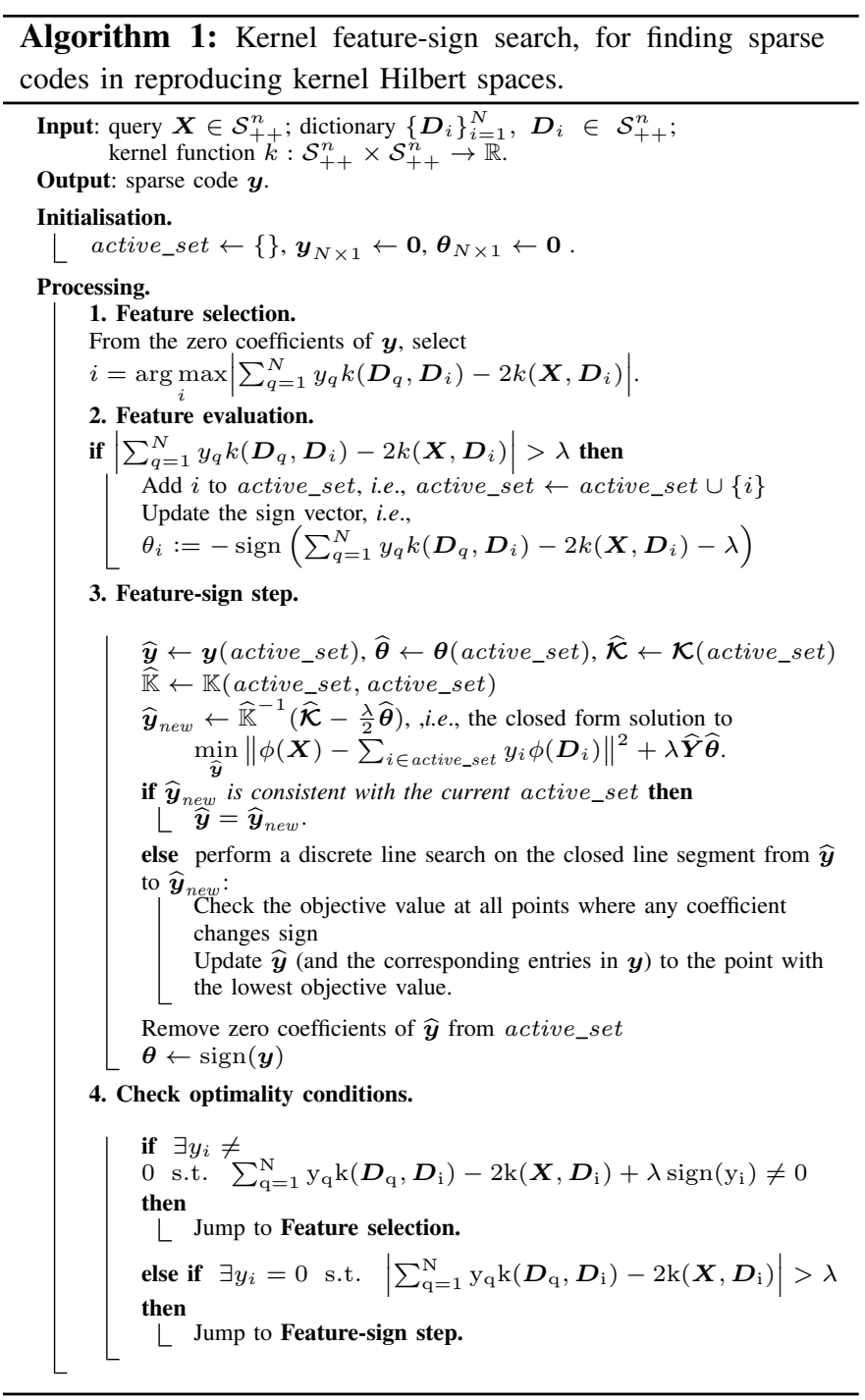

Given the objective function defined in Eqn. (12), if the signs (positive, zero, or negative) of the $y_{j}$ are known at the optimal value, each term of $\|\boldsymbol{y}\|_{1}=\sum_{j=1}^{N}\left|y_{i}\right|$ can be replaced by either $y_{i},-y_{i}$ or 0 . Considering only non-zero coefficients, this reduces Eqn. (12) to a standard, unconstrained quadratic optimization problem (QP) [6], which has an analytic solution.

The Feature-Sign Algorithm comprises of four basic steps. The first two steps can be considered as a greedy search. The search is directed towards selecting a new feature that maximizes the rate of decrements in Eqn. (12). This is accomplished by computing the first order derivative of Eqn. (12) with respect to features, i.e.,

$$
\frac{\partial}{\partial y_{j}} l_{\phi}(\boldsymbol{X}, \mathbb{D})=\sum_{q=1}^{N} y_{q} k\left(\boldsymbol{D}_{q}, \boldsymbol{D}_{j}\right)-2 k\left(\boldsymbol{X}, \boldsymbol{D}_{j}\right)+\lambda .
$$

Since an estimate of the feature signs is available, in the third step (feature-sign step) it is possible to find the solution of the unconstrained QP of $\min _{\widehat{\boldsymbol{y}}} \widetilde{f}(\widehat{\boldsymbol{y}})$, where

$$
\begin{aligned}
\tilde{f}(\widehat{\boldsymbol{y}}) & =\left\|\phi(\boldsymbol{X})-\sum_{i \in \text { active_set }} y_{i} \phi\left(\boldsymbol{D}_{i}\right)\right\|^{2}+\lambda \widehat{\boldsymbol{\theta}}^{T} \widehat{\boldsymbol{y}} \\
& =k(\boldsymbol{X}, \boldsymbol{X})-2 \widehat{\boldsymbol{y}}^{T} \widehat{\mathcal{K}}+\widehat{\boldsymbol{y}}^{T} \widehat{\mathbb{K}} \widehat{\boldsymbol{y}}+\lambda \widehat{\boldsymbol{\theta}}^{T} \widehat{\boldsymbol{y}}
\end{aligned}
$$

In Eqn. (31), $\widehat{\boldsymbol{y}}=\boldsymbol{y}$ (active_set), $\widehat{\boldsymbol{\theta}}=\boldsymbol{\theta}$ (active_set) and $\widehat{\mathcal{K}}=\mathcal{K}$ (active_set) are subvectors corresponding to active_set. Similarly, $\widehat{\mathbb{K}}=\mathbb{K}$ (active_set, active_set) is a submatrix corresponding to active_set with active_set being the subset of selected features. The closed form solution, i.e., $\widehat{\mathbb{K}}^{-1}\left(\widehat{\mathcal{K}}-\frac{\lambda \widehat{\boldsymbol{\theta}}}{2}\right)$, can be derived by computing the root of first order derivative of Eqn. (31) with respect to $\widehat{\boldsymbol{y}}$. The final step of the algorithm is an optimality check to verify that the features and the corresponding signs are truly consistent with the objective function in Eqn. (12).

The convergence of the feature sign algorithm has been discussed for Euclidean spaces in [31] and can be readily extended to the kernelized case.

\section{ACKNOWLEDGEMENTS}

NICTA is funded by the Australian Government as represented by the Department of Broadband, Communications and the Digital Economy, as well as the Australian Research Council (ARC) through the ICT Centre of Excellence program.

\section{REFERENCES}

[1] M. Aharon, M. Elad, and A. Bruckstein. K-SVD: An algorithm for designing overcomplete dictionaries for sparse representation. IEEE Transactions on Signal Processing, 54(11):4311-4322, Nov. 2006.

[2] L. Bazzani, M. Cristani, and V. Murino. Symmetry-driven accumulation of local features for human characterization and re-identification Computer Vision and Image Understanding, 117(2):130-144, 2013.

[3] C. Berg, J. P. R. Christensen, and P. Ressel. Harmonic Analysis on Semigroups. Springer, 1984.

[4] R. Bhatia. Positive Definite Matrices. Princeton University Press, 2007.

[5] C. M. Bishop. Pattern Recognition and Machine Learning. Springer, Heidelberg, 2006

[6] S. Boyd and L. Vandenberghe. Convex Optimization. Cambridge University Press, New York, 2004.

[7] R. Caseiro, J. F. Henriques, P. Martins, and J. Batista. Semi-intrinsic mean shift on Riemannian manifolds. In Proceedings of European Conference on Computer Vision (ECCV), pages 342-355, Florence, Italy, Oct. 2012.

[8] D. Chen, X. Cao, F. Wen, and J. Sun. Blessing of dimensionality: Highdimensional feature and its efficient compression for face verification. In Proceedings of IEEE Conference on Computer Vision and Pattern Recognition (CVPR), pages 3025-3032, Portland, OR, Jun. 2013.

[9] Y. Chen, E. K. Garcia, M. R. Gupta, A. Rahimi, and L. Cazzanti. Similarity-based classification: Concepts and algorithms. Journal of Machine Learning Research, 10:747-776, Mar. 2009.

[10] A. Cherian, S. Sra, A. Banerjee, and N. Papanikolopoulos. JensenBregman LogDet divergence with application to efficient similarity search for covariance matrices. IEEE Transactions on Pattern Analysis and Machince Intelligence, 35(9):2161-2174, Sep. 2013.

[11] N. Dalal and W. Triggs. Histograms of oriented gradients for human detection. In Proceedings of IEEE Conference on Computer Vision and Pattern Recognition (CVPR), pages 886-893, San Diego, CA, Jun. 2005.

[12] A. P. Dempster, N. M. Laird, and D. B. Rubin. Maximum likelihood from incomplete data via the EM algorithm. Journal of the Royal Statistical Society. Series B (Methodological), 39(1):1-38, 1977.

[13] M. Elad. Sparse and Redundant Representations: From Theory to Applications in Signal and Image Processing. Springer, Heidelberg, 2010.

[14] A. Ess, B. Leibe, and L. V. Gool. Depth and appearance for mobile scene analysis. In Proceedings of International Conference on Computer Vision (ICCV), pages 1-8, Rio de Janeiro, Brazil, Oct. 2007.

[15] M. Faraki, M. Palhang, and C. Sanderson. Log-Euclidean bag of words for human action recognition. IET Computer Vision, (in press). http://dx.doi.org/10.1049/iet-cvi.2014.0018.

[16] S. Gao, I. W.-H. Tsang, and L.-T. Chia. Kernel sparse representation for image classification and face recognition. In Proceedings of European Conference on Computer Vision (ECCV), volume IV, pages 1-14, Heraklion, Crete, Greece, Sep. 2010. 
[17] M. Grant and S. Boyd. CVX: Matlab software for disciplined convex programming, version 2.0 beta. http://cvxr.com/cvx.

[18] K. Guo, P. Ishwar, and J. Konrad. Action recognition from video using feature covariance matrices. IEEE Transactions on Image Processing, 22(6):2479-2494, Jun. 2013.

[19] M. Harandi, C. Sanderson, C. Shen, and B. Lovell. Dictionary learning and sparse coding on Grassmann manifolds: An extrinsic solution. In Proceedings of International Conference on Computer Vision (ICCV), pages 3120-3127, Sydney, Australia, Dec. 2013.

[20] M. T. Harandi, M. Salzmann, and R. Hartley. From manifold to manifold: Geometry-aware dimensionality reduction for SPD matrices. In Proceedings of European Conference on Computer Vision (ECCV), pages 17-32, Zurich, Switzerland, Sep. 2014.

[21] M. T. Harandi, C. Sanderson, R. Hartley, and B. C. Lovell. Sparse coding and dictionary learning for symmetric positive definite matrices: A kernel approach. In Proceedings of European Conference on Computer Vision (ECCV), pages 216-229, Florence, Italy, Oct. 2012.

[22] M. T. Harandi, C. Sanderson, A. Wiliem, and B. C. Lovell. Kerne analysis over Riemannian manifolds for visual recognition of actions, pedestrians and textures. In IEEE Workshop on Applications of Computer Vision (WACV), pages 433-439, Breckenridge, CO, Jan. 2012.

[23] M. Hein and O. Bousquet. Hilbertian metrics and positive definite kernels on probability measures. In Proceedings of Int. Conf. Artificial Intelligence \& Statistics, pages 136-143, Barbados, Jan. 2005.

[24] N. J. Higham. Computing a nearest symmetric positive semidefinite matrix. Linear Algebra and its Applications, 103:103-118, May 1988.

[25] J. Ho, Y. Xie, and B. Vemuri. On a nonlinear generalization of sparse coding and dictionary learning. In Proceedings of International Conference on Machine Learning (ICML), pages 1480-1488, Atlanta, Jun. 2013.

[26] M. E. Hussein, M. Torki, M. A. Gowayyed, and M. El-Saban. Human action recognition using a temporal hierarchy of covariance descriptors on 3D joint locations. In Proceedings of International Joint Conference on Artificial Intelligence (IJCAI), pages 2466-2472, Beijing, China, Aug. 2013.

[27] S. Jayasumana, R. Hartley, M. Salzmann, H. Li, and M. Harandi. Kernel methods on the Riemannian manifold of symmetric positive definite matrices. In Proceedings of IEEE Conference on Computer Vision and Pattern Recognition (CVPR), pages 73-80, Portland, OR, Jun. 2013.

[28] A. Kovashka and K. Grauman. Learning a hierarchy of discriminative space-time neighborhood features for human action recognition. In Proceedings of IEEE Conference on Computer Vision and Pattern Recognition (CVPR), pages 2046-2053, San Francisco, CA, Jun. 2010.

[29] B. Kulis, M. A. Sustik, and I. S. Dhillon. Low-rank kernel learning with Bregman matrix divergences. Journal of Machine Learning Research, 10:341-376, Feb. 2009.

[30] H. Lee. Unsupervised Feature Learning via Sparse Hierarchical Representations. Ph.D. Thesis, Department of Computer Science, Stanford University, 2010.

[31] H. Lee, A. Battle, R. Raina, and A. Y. Ng. Efficient sparse coding algorithms. In B. Schölkopf, J. Platt, and T. Hoffman, editors, Advances in Neural Information Processing Systems 19, pages 801-808. MIT Press, 2007.

[32] J. M. Lee. Introduction to Smooth Manifolds. Graduate Texts in Mathematics (Book 218). Springer, Heidelberg, 2012.

[33] Z. Liao, J. Rock, Y. Wang, and D. Forsyth. Non-parametric filtering for geometric detail extraction and material representation. In Proceedings of IEEE Conference on Computer Vision and Pattern Recognition (CVPR), pages 963-970, Portland, OR, Jun. 2013.

[34] C. Liu, L. Sharan, E. H. Adelson, and R. Rosenholtz. Exploring features in a Bayesian framework for material recognition. In Proceedings of IEEE Conference on Computer Vision and Pattern Recognition (CVPR), pages 239-246, San Francisco, CA, Jun. 2010.

[35] D. G. Lowe. Distinctive image features from scale-invariant keypoints. International Journal of Computer Vision, 60(2):91-110, 2004.

[36] P. J. Moreno, P. P. Ho, and N. Vasconcelos. A Kullback-Leibler divergence based kernel for SVM classification in multimedia applications. In S. Thrun, L. Saul, and B. Schölkopf, editors, Advances in Neural Information Processing Systems 16, pages 1385-1392. MIT Press, 2004.

[37] M. Müller, T. Röder, M. Clausen, B. Eberhardt, B. Krüger, and A. Weber. Documentation: Mocap database HDM05. Technical Report CG-2007-2, Universität Bonn, 2007.

[38] H. Nguyen, V. Patel, N. Nasrabadi, and R. Chellappa. Design of nonlinear kernel dictionaries for object recognition. IEEE Transactions on Image Processing, 22(12):5123-5135, Dec. 2013.

[39] B. A. Olshausen and D. J. Field. Emergence of simple-cell receptive field properties by learning a sparse code for natural images. Nature, 381(6583):607-609, 1996

[40] Y. Pang, Y. Yuan, and X. Li. Gabor-based region covariance matrices for face recognition. IEEE Transactions on Circuits and Systems for Video Technology, 18(7):989-993, Jul. 2008.

[41] X. Pennec, P. Fillard, and N. Ayache. A Riemannian framework for tensor computing. International Journal of Computer Vision, 66(1):4166, 2006.

[42] P. Phillips, H. Moon, S. Rizvi, and P. Rauss. The FERET evaluation methodology for face-recognition algorithms. IEEE Transactions on Pattern Analysis and Machince Intelligence, 22(10):1090-1104, Oct. 2000.

[43] T. Randen and J. H. Husøy. Filtering for texture classification: A comparative study. IEEE Transactions on Pattern Analysis and Machince Intelligence, 21(4):291-310, Apr. 1999.

[44] M. Rodriguez, J. Ahmed, and M. Shah. Action MACH a spatio-temporal maximum average correlation height filter for action recognition. In Proceedings of IEEE Conference on Computer Vision and Pattern Recognition (CVPR), pages 1-8, Anchorage, AK, Jun. 2008.

[45] A. Sanin, C. Sanderson, M. Harandi, and B. Lovell. Spatio-temporal covariance descriptors for action and gesture recognition. In Proceedings of IEEE Workshop on Applications of Computer Vision (WACV), pages 103-110, Clearwater Beach, FL, Jan. 2013.

[46] B. Schölkopf. The kernel trick for distances. In T. Leen, T. Dietterich, and V. Tresp, editors, Advances in Neural Information Processing Systems 13, pages 301-307. MIT Press, 2001.

[47] W. R. Schwartz and L. S. Davis. Learning discriminative appearancebased models using partial least squares. In Brazilian Symposium on Computer Graphics and Image Processing, pages 322-329, Rio de Janeiro, Brazil, Oct. 2009.

[48] L. Sharan, R. Rosenholtz, and E. Adelson. Material perception: What can you see in a brief glance? Journal of Vision, 9(8):784-784, 2009.

[49] J. Shawe-Taylor and N. Cristianini. Kernel Methods for Pattern Analysis Cambridge University Press, New York, 2004.

[50] R. Sivalingam, D. Boley, V. Morellas, and N. Papanikolopoulos. Positive definite dictionary learning for region covariances. In Proceedings of International Conference on Computer Vision (ICCV), pages 1013-1019, Barcelona, Spain, Nov. 2011.

[51] R. Sivalingam, D. Boley, V. Morellas, and N. Papanikolopoulos. Tensor sparse coding for positive definite matrices. IEEE Transactions on Pattern Analysis and Machince Intelligence, 36(3):592-605, Mar. 2014.

[52] S. Sra. A new metric on the manifold of kernel matrices with application to matrix geometric means. In P. Bartlett, editor, Advances in Neural Information Processing Systems 25, pages 144-152. Curran Associates, 2013.

[53] S. Sra and A. Cherian. Generalized dictionary learning for symmetric positive definite matrices with application to nearest neighbor retrieval. In Proceedings of European Conference on Machine Learning, pages 318-332, Athens, Greece, Sep. 2011.

[54] O. Tuzel, F. Porikli, and P. Meer. Region covariance: A fast descriptor for detection and classification. In Proceedings of European Conference on Computer Vision (ECCV), pages 589-600, Graz, Austria, May 2006.

[55] O. Tuzel, F. Porikli, and P. Meer. Pedestrian detection via classification on Riemannian manifolds. IEEE Transactions on Pattern Analysis and Machince Intelligence, 30(10):1713-1727, Oct. 2008.

[56] M. Varma and A. Zisserman. A statistical approach to material classification using image patch exemplars. IEEE Transactions on Pattern Analysis and Machince Intelligence, 31(11):2032-2047, Nov. 2009.

[57] H. Wang, M. M. Ullah, A. Kläser, I. Laptev, and C. Schmid. Evaluation of local spatio-temporal features for action recognition. In British Machine Vision Conference (BMVC), London, UK, Sep. 2009.

[58] Z. Wang and B. C. Vemuri. An affine invariant tensor dissimilarity measure and its applications to tensor-valued image segmentation. In Proceedings of IEEE Conference on Computer Vision and Pattern Recognition (CVPR), volume 1, pages 228-233, Washington, DC, Jun. 2004.

[59] J. Wright, A. Yang, A. Ganesh, S. Sastry, and Y. Ma. Robust face recognition via sparse representation. IEEE Transactions on Pattern Analysis and Machince Intelligence, 31(2):210-227, Feb. 2009.

[60] X. Wu, D. Xu, L. Duan, and J. Luo. Action recognition using context and appearance distribution features. In Proceedings of IEEE Conference on Computer Vision and Pattern Recognition (CVPR), pages 489-496, Providence, RI, Jun. 2011.

[61] M. Yang and L. Zhang. Gabor feature based sparse representation for face recognition with Gabor occlusion dictionary. In Proceedings of European Conference on Computer Vision (ECCV), volume IV, pages 448-461, Heraklion, Crete, Greece, Sep. 2010.

[62] C. Yuan, W. Hu, X. Li, S. Maybank, and G. Luo. Human action recognition under log-Euclidean Riemannian metric. In Proceedings of Asian Conference on Computer Vision (ACCV), pages 343-353. Xi'an, China, Sep. 2009. 


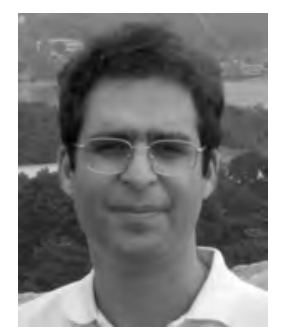

Mehrtash Harandi received the BSc in Electronics from Sharif University of technology, MSc and $\mathrm{PhD}$ degrees in computer science from the University of Tehran, Iran. He is a senior researcher at Computer Vision Research Group, NICTA, Australia. His main research interests are theoretical and computational methods in computer vision and machine learning with a focus on Riemannian geometry.

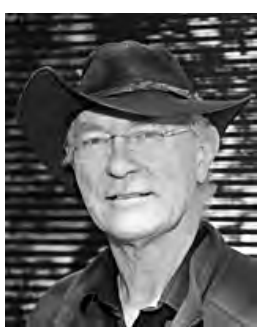

Richard Hartley is a member of the computer vision group in the Research School of Engineering, Australian National University, where he has been since January, 2001. He is also a member of the computer vision research group in NICTA. Dr. Hartley worked at the General Electric Research and Development Center from 1985 to 2001, working first in VLSI design, and later in computer vision. He became involved with Image Understanding and Scene Reconstruction working with GE's Simulation and Control Systems Division. He is an author (with Andrew Zisserman) of the book Multiple View Geometry in Computer Vision.

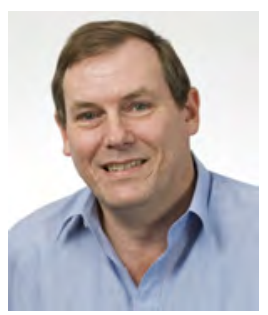

Brian Lovell received the BEng in electrical engineering in 1982, the BSc in computer science in 1983, and the $\mathrm{PhD}$ in signal processing in 1991: all from the University of Queensland (UQ), Australia. Professor Lovell was Research Leader at NICTA and Research Director of the Surveillance Research group at UQ. He serves on the Editorial Board of Pattern Recognition Letters and reviews for many of the major journals in Computer Vision.

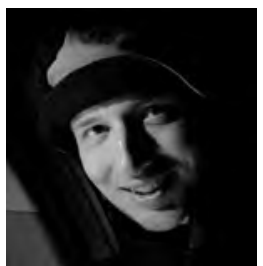

Conrad Sanderson is a Research Leader at NICTA, Australia. He received the $\mathrm{PhD}$ degree in 2003 from Griffith University, and the MBA degree in 2012 from the University of Queensland. He has worked on speech recognition and language translation at the Advanced Telecommunication Research Laboratories (Japan), audio-visual biometrics at the IDIAP Research Institute (Switzerland), computer vision for military applications at the University of Adelaide (Australia), as well as natural language processing, bioinformatics and automated surveillance at NICTA. His work on innovative surveillance technologies has led to several industry awards. He has served as a reviewer for major international conferences and scientific journals. His current interests are in the adaptation and commercialisation of research outcomes towards industrial use, as well as machine learning, pattern recognition, and predictive analytics. 